\title{
HOMOTOPY SIMULATION OF NON-NEWTONIAN SPRIGGS FLUID FLOW OVER A FLAT PLATE WITH OSCILLATING MOTION
}

\author{
A.K. RAY and B. VASU \\ Department of Mathematics, Motilal Nehru National Institute of Technology \\ Allahabad, INDIA \\ E-mail: bvasu@mnnit.ac.in; rma0215@mnnit.ac.in \\ R.S.R. GORLA* \\ Department of Mechanical Engineering, Cleveland State University \\ Ohio, USA \\ E-mail: r.gorla@csuohio.edu
}

\begin{abstract}
An incompressible flow of a non-Newtonian Spriggs fluid over an unsteady oscillating plate is investigated using the Homotopy Analysis Method (HAM). An analytic solution of sine and cosine oscillations of the plate has been obtained. The similarity transformation is introduced to reduce the governing partial differential equations into a single non-linear dimensionless partial differential equation. The effects of the power index of Spriggs fluid and convergence control parameter of HAM for the flow are studied extensively. The range of the convergence control parameter for convergence of series solution for different values of the power index of Spriggs fluid is obtained. The solution for a Spriggs fluid is noticeably different from the solution obtained for a Newtonian fluid. The influences of the shear thinning and shear thickening fluid on the velocity profile are shown graphically. The transient flow effect is higher for non-Newtonian Spriggs fluid than that of a Newtonian fluid. It is also observed that the interval to reach the steady state for the cosine case is less than the sine case. The applications of Stokes' second problem have been widely found in the variety of fields of biomedical, medical, chemical, micro and nanotechnology.
\end{abstract}

Key words: incompressible flow, oscillatory plate, Stokes' second problem, HAM, non-Newtonian Spriggs fluid.

\section{Introduction}

The study of characteristics of a non-Newtonian fluid flow is very essential because they have important applications in the vast field of industry, science and engineering. Non-Newtonian nature of fluids has been studied by many models [1-2]. Spriggs fluid is one of such non-Newtonian fluid models and it is a Generalized Newtonian fluid (GNF) [3-4]. Spriggs fluid, also called truncated power law fluid, was first suggested by Spriggs [5] in 1965. At low and high shear rates, the power law model does not predict any Newtonian plateau and gives inaccurate results, whereas the constitutive equation of Spriggs fluid truncates the region with the reference shear rate before which the fluid will behave like Newtonian and after which it performs like a power law fluid model. Recently, Lavrov [6] explained the hydraulic fracturing application of flow of Spriggs fluid between parallel walls and concluded that the Spriggs model increases the accuracy of hydraulic fracturing simulation, as compared to the power law model. Spriggs fluid as a shear thinning fluid can be very useful in study on elastohydrodynamic film thickness [4-7]. Adusmilli and Hill [8] investigated the transient laminar flow of Spriggs fluid.

The study on the flow of non-Newtonian fluids over a plate has attracted attention of many researchers [9-13]. Stokes' first and second problems are one of those types of flows. Stokes' first problem [14-18] refers the sudden start of plane plate. The plate moves with a constant velocity tangentially to its own plane, whereas Stokes' second problem is the periodic motion of a flat plate. Rajagopal [19] gave the first

\footnotetext{
* To whom correspondence should be addressed
} 
exact solution of Stokes' second problem for non-Newtonian fluids. Recently, Ishfaq et al. [20] investigated Stokes' second problem for nanofluids. Duan and Qiu [21] provided periodic solution of Stokes' second problem for viscoelastic fluids. Semi -analytic methods such as the Adomain Decomposition Method (ADM), Homotopy Perturbation Method (HPM), Variational Iteration Method (VIM) were used to investigate the fluid behaviour over a flat plate with oscillating motion and wall transpiration by Farkhadnia et al. [22]. Oscillating flow of an incompressible magnetohydrodynamic (MHD) second grade fluid in a porous half space was investigated by Ali et al. [23]. An analytic solution of second problem of Stokes' for a second grade fluid using the Laplace transform and perturbation technique was given by Asghar et al. [24]. Fetecau et al. [25] used the Laplace transformation to give exact solution of Stokes' second problem for a Newtonian fluid. Ai and Vafai [26] considered eight non-Newtonian models and compared velocity and temperature profiles of these models with Newtonian fluids. The analytic solution of Stokes' problem using the Laplace transform method was studied in depth by Erdogan [27] and the solution includes both steady and transient solutions.

The governing equations for fluid mechanics problems such as Stokes' second a difficult task to get analytical approximations of such non-linear partial differential equations. Traditionally, perturbation methods [28, 29], have been widely applied to give analytic approximations of non-linear problems but, unfortunately, they rely highly on small/large parameters and these parameters restricts-the analysis because many non-linear problems do not contain such small/large parameters. An analytic method known as the Homotopy Analysis Method which is based on the topological concept "Homotopy" has been developed by Liao [30]. The method does not depend on any small/large parameter. HAM also provides a suitable way to control the convergence of series solution by using a parameter called the convergence control parameter. Due to these reasons, HAM is valid for both weak as well as strong non-linear problems. HAM is a powerful method and is applied to many problems nowadays. Recently, Rashidi et al. [31] discussed the mixed convection boundary layer flow of a micro polar fluid towards a heated shrinking sheet by using HAM. A non-linear convective stagnation point flow of a thixotropic fluid towards a stretching sheet within the frame of Catteneo Christov heat flux theory was studied by Hayat et al. [32]. A non-linear fractional differential equation was solved by Das et al. [33] using four different methods, viz., HAM, VIM, ADM and Power series method (PSM). An automatic scheme was coupled with HAM to solve non-linear algebraic equations by Al-Shara et al. [34]. HAM is very efficient and applied to many non-linear problems [35-39].

Many researchers have used different non-Newtonian models to study and investigate the flow for Stokes' second problem. In this paper, the behaviour of Spriggs fluid over a flat plate is presented where the plate is initially at rest and then starts oscillating. Similarity transformation is used to investigate the flow. The transformed non-dimensionalised partial differential equation after using similarity transformation is solved by HAM. The plate is subjected to both sine and cosine oscillations. Variations of the velocity profile are presented graphically for different values of the convergence control parameter of HAM and power index of Spriggs fluid. Also, the effect of distance from the wall and the power law index over wall shear stress is discussed and presented graphically. It is found that velocity decays to zero, faster for a non-Newtonian fluid than for a Newtonian fluid. Velocity dies away early for a shear thickening fluid than for a shear thinning fluid.

The paper is organized as follows: Section 2 contains a description of the problem and its mathematical modelling. Section 3 provides the solution of the problem via HAM for boundary condition subjected to both sine and cosine oscillations. Section 4 includes the graphical representation of velocity and wall shear stress for different parameters, such as the convergence control parameter, distance from the wall and power index. Finally, Section 5 concludes the results of Section 2, 3 and 4.

\section{Mathematical modelling}

\subsection{Governing equation}

Consider a two dimensional boundary layer unsteady viscous flow in an infinite fluid medium. The infinite flat plate lies on the $x$ - axis at time $t=0$ shown in Fig.1a. For $t>0$, the plate starts moving with velocity $U_{w}(x)$, shown in Fig. $1 \mathrm{~b}$. 
Let $U_{w}(x)=U \operatorname{Sin} \omega t$ denote the speed of the flat plate.

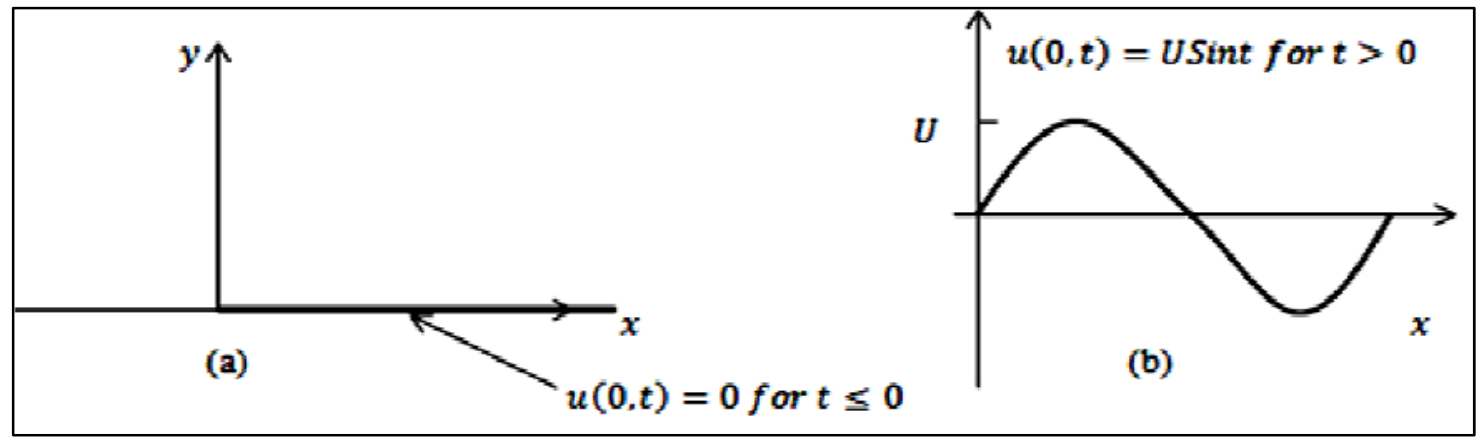

Fig.1. Schematic diagram and co-ordinate system for Sine oscillation.

Modelling of non-Newtonian Spriggs fluid flow for Stokes' second problem in the absence of body force is given below:

The equations which governs the flow are given by

- continuity equation

$$
\frac{\partial u}{\partial x}+\frac{\partial v}{\partial y}=0
$$

- momentum equations

$$
\begin{gathered}
\rho\left[\frac{\partial u}{\partial t}+u \frac{\partial u}{\partial x}+v \frac{\partial u}{\partial y}\right]=-\frac{\partial p}{\partial x}+\frac{\partial \tau_{x x}}{\partial x}+\frac{\partial \tau_{x y}}{\partial x}, \\
\rho\left[\frac{\partial v}{\partial t}+u \frac{\partial v}{\partial x}+v \frac{\partial v}{\partial y}\right]=-\frac{\partial p}{\partial y}+\frac{\partial \tau_{y x}}{\partial y}+\frac{\partial \tau_{y y}}{\partial y} .
\end{gathered}
$$

Here $u$ and $v$ are velocity components in the direction of $x$ and $y$ respectively, $t$ is time, $\rho$ is the density of fluid, $p$ is pressure and $U$ denotes representative velocity. As the plate starts moving, it oscillates parallel to its plane with velocity $U \operatorname{Sin} \omega t$ or $U \operatorname{Cos} \omega t$. Under these conditions, no flow occurs in the $y$ direction and the flow velocity $u$ at a given point depends only on its $y$-coordinate and time i.e. $u=u(y, t)$

$$
v=0, \quad \frac{\partial u}{\partial x}=0 .
$$

Stress components using Eqs (2.4) are

$$
\tau_{y x}=\tau_{y y}=\tau_{x x}=0 .
$$

Using Eqs (2.4) and (2.5), $y$-momentum Eq.(2.3) implies

$$
\frac{\partial p}{\partial y}=0 .
$$

As pressure is independent of $x$ 


$$
\frac{\partial p}{\partial x}=0
$$

so Eqs (2.6) and (2.7) imply that pressure is constant.

The viscosity function of the truncated power law of Spriggs [2] is defined as

$$
\eta=\left\{\begin{array}{cc}
\eta_{0} ; & \dot{\gamma} \leq \dot{\gamma}_{0} \\
\eta_{0}\left(\frac{\dot{\gamma}}{\dot{\gamma}_{0}}\right)^{n-1} ; & \dot{\gamma} \geq \dot{\gamma}_{0}
\end{array}\right.
$$

where $\eta_{0}, \dot{\gamma}$ and $\dot{\gamma}_{0}$ are dynamic viscosity, shear rate and reference shear rate, respectively. $\dot{\gamma}_{0}$ is the value of $\dot{\gamma}$ from where shear thinning behaviour begins. The stress component $\tau_{x y}$ for a non-Newtonian Spriggs fluid is

$$
\tau_{x y}=\left\{\begin{array}{cc}
\eta_{0} \frac{\partial u}{\partial y} & ; \dot{\gamma} \leq \dot{\gamma}_{0} \\
\eta_{0}\left(\frac{\dot{\gamma}}{\dot{\gamma}_{0}}\right)^{n-1} \frac{\partial u}{\partial y} ; \dot{\gamma} \geq \dot{\gamma}_{0}
\end{array}\right.
$$

Using Eqs (2.4), (2.5), (2.7) and (2.9), $x$-momentum Eq.(2.2) reduces to a partial differential equation

$$
\rho \frac{\partial u}{\partial t}=\eta_{0} \begin{cases}\frac{\partial^{2} u}{\partial y^{2}} & \dot{\gamma} \leq \dot{\gamma}_{0} \\ \frac{1}{\left(\dot{\gamma}_{0}\right)^{n-1}} \frac{\partial}{\partial y}\left(\frac{\partial u}{\partial y}\right)^{n} ; & \dot{\gamma} \geq \dot{\gamma}_{0}\end{cases}
$$

and the respective boundary condition will be

$$
\begin{aligned}
& u(y, 0)=0 \quad \text { for } \quad y>0, \\
& u(0, t)=\left\{\begin{array}{ccc}
0 & ; & t \leq 0 \\
U \operatorname{Sin} \omega t & ; & t \geq 0
\end{array}\right. \\
& u(y, t) \rightarrow 0 \quad \text { as } \quad y \rightarrow \infty .
\end{aligned}
$$

\subsection{Dimensionless governing equation}

We introduce the following similarity transformation according to Ali and Vafai [26]

$$
f(\eta)=\frac{u}{U}, \quad \tau=\omega t, \quad \text { and } \quad \eta=y\left(\frac{\omega}{v_{0}}\right)^{1 / 2}
$$

where $U$ is the reference velocity, $\omega$ is frequency and $v_{0}$ is representative viscosity.

Therefore 


$$
\begin{aligned}
& \frac{\partial u}{\partial t}=U \omega \frac{\partial f}{\partial \tau}, \\
& \dot{\gamma}=\frac{\partial u}{\partial y}=U\left(\frac{\omega}{v_{0}}\right)^{1 / 2} \frac{\partial f}{\partial \eta}=\dot{\gamma}_{0} \frac{\partial f}{\partial \eta} .
\end{aligned}
$$

Similarly,

$$
\frac{\partial^{2} u}{\partial y^{2}}=\frac{U \omega}{v_{0}} \frac{\partial^{2} f}{\partial \eta^{2}}
$$

Substitute these values, i.e. $\frac{\partial u}{\partial t}, \frac{\partial u}{\partial y}$ and $\frac{\partial^{2} u}{\partial y^{2}}$ into Eq.(2.10). Then it yields

$$
\begin{aligned}
& \rho\left[U \omega \frac{\partial f}{\partial \tau}\right]=\eta_{0} \begin{cases}\frac{U \omega}{v_{0}} \frac{\partial^{2} f}{\partial \eta^{2}} \\
\frac{n U^{n-1}}{\left(\dot{\gamma}_{0}\right)^{n-1}}\left(\frac{\omega}{v_{0}}\right)^{(n-1) / 2}\left(\frac{\partial f}{\partial \eta}\right)^{n-1} \frac{U \omega}{v_{0}} \frac{\partial^{2} f}{\partial \eta^{2}} ; & \frac{\partial f}{\partial \eta} \geq 1\end{cases} \\
& \frac{\partial f}{\partial \tau}= \begin{cases}\frac{\partial^{2} f}{\partial \eta^{2}} & \frac{\partial f}{\partial \eta} \leq 1 \\
n\left(\frac{\partial f}{\partial \eta}\right)^{n-1} \frac{\partial^{2} f}{\partial \eta^{2}} ; & \frac{\partial f}{\partial \eta} \geq 1\end{cases}
\end{aligned}
$$

where $\dot{\gamma}_{0}=U\left(\frac{\omega}{v_{0}}\right)^{1 / 2}$ is the reference shear rate [26] which denotes the magnitude of wall shear rate as a combined effect of $U, v_{0}$ and $\omega$.

Case I: $\dot{\gamma} \leq \dot{\gamma}_{0}$, i.e. $\frac{\partial f}{\partial \eta} \leq 1$

From Eq.(2.13) we have

$$
\frac{\partial f}{\partial \tau}=\frac{\partial^{2} f}{\partial \eta^{2}}
$$

Case II: $\dot{\gamma} \geq \dot{\gamma}_{0}$, i.e. $\frac{\partial f}{\partial \eta} \geq 1$

We have from Eq.(2.13)

$$
\frac{\partial f}{\partial \tau}-n\left(\frac{\partial f}{\partial \eta}\right)^{n-1} \frac{\partial^{2} f}{\partial \eta^{2}}=0
$$



follows

Initial and boundary conditions i.e., Eqs (2.11a-c), after introducing (2.12), are transformed as

$$
f(\eta, 0)=0, \quad f(0, \tau)=\operatorname{Sin} \tau \quad \text { and } \quad f(\infty, \tau)=0 .
$$

Note that for $n=1$, both Eqs (2.14) and (2.15) became identical.

If the principal boundary condition (Fig.2) is

$$
u(0, t)=\left\{\begin{array}{ccc}
0 & ; & t \leq 0 \\
U \operatorname{Cos} \omega t ; & t \geq 0
\end{array}\right.
$$

then the respective non-dimensionalised boundary condition becomes

$$
f(\eta, 0)=0, \quad f(0, \tau)=\operatorname{Cos} \tau \quad \text { and } \quad f(\infty, \tau)=0 .
$$

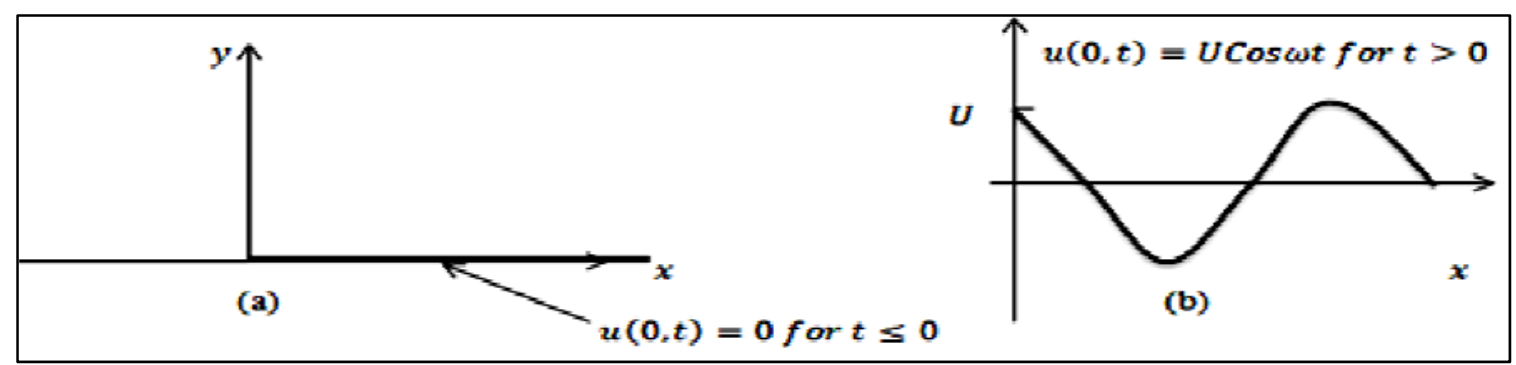

Fig.2. Schematic diagram and co-ordinate system for Stokes' second problem for cosine oscillation.

\section{Analytic approximation by HAM}

An analytic solution for non-dimensionalised partial differential Eqs (2.14) and (2.15) with initial and boundary conditions in Eqs (2.16) and (2.17) have two cases, the first case is for a Newtonian fluid and the other case is for a non-Newtonian power law fluid as per definition of Spriggs fluid. Both the cases are discussed below.

3.1. Case I: for $\frac{\partial f}{\partial \eta} \leq 1$

The exact solution for Eq.(2.14) with respect to the initial and boundary conditions in Eqs (2.16) and (2.17) is given by

$$
\begin{aligned}
& u(y, t)=U \exp \left(-\sqrt{\frac{n}{2 \mathrm{v}_{0}}} y\right) \operatorname{Sin}\left(n t-\sqrt{\frac{n}{2 \mathrm{v}_{0}}} y\right), \\
& u(y, t)=U \exp \left(-\sqrt{\frac{n}{2 \mathrm{v}_{0}}} y\right) \operatorname{Cos}\left(n t-\sqrt{\frac{n}{2 \mathrm{v}_{0}}} y\right),
\end{aligned}
$$

Eqs (3.1) and (3.2) are the exact solutions for the flow of a Navier-Stokes fluid [40] past a plate with oscillatory motion (sine and cosine oscillation respectively). 
3.2. Case II: for $\frac{\partial f}{\partial \eta} \geq 1$

An analytic solution for Eq.(2.15) with respect to the initial and boundary conditions in Eqs (2.16) and (2.17) is obtained by HAM. An initial guess $f_{0}$, auxiliary linear operator $\mathcal{L}$, non-linear operator $N$, convergence control parameter $c_{0}$ are properly chosen to get the convergent homotopy series solution.

On the basis of initial and boundary conditions, initial guess $f_{0}$ is taken as

$$
f_{0}(\eta, \tau)=e^{-\eta} \operatorname{Sin} \tau
$$

and the linear operator as

$$
\mathcal{L}(f)=f^{\prime \prime}-f,
$$

with the property that

$$
\mathcal{L}\left(a_{1} e^{-\eta}+a_{2} e^{\eta}\right)=0
$$

Using Eq.(2.15) we define the non-linear operator as

$$
\mathcal{N}[\phi(\eta, \tau ; q)]=\frac{\partial \phi}{\partial \tau}-n\left(\frac{\partial \phi}{\partial \eta}\right)^{n-1} \frac{\partial^{2} \phi}{\partial \eta^{2}} .
$$

Zeroth order deformation equation

$$
(1-q) \mathcal{L}\left[\phi(\eta, \tau ; q)-f_{0}(\eta, \tau)\right]=c_{0} q \mathcal{N}[(\phi(\eta, \tau ; q))]
$$

subject to the boundary condition

$$
\phi(0, \tau ; q)=\operatorname{Sin} \tau \quad \text { and } \quad \phi(\infty, \tau ; q) \rightarrow 0 .
$$

Note that $f_{0}(\eta, \tau)$ satisfies the conditions in Eqs (2.16) and (2.17) as well as the zeroth order deformation Eq.(3.7). For $q=0, \phi(\eta, \tau ; 0)=f_{0}(\eta, \tau)$ and for $q=1$, since $c_{0} \neq 0$, Eq.(3.7) is equivalent to Eqs (2.14) and (2.15), provided $\phi(\eta, \tau ; 1)=f(\eta, \tau)$.

The series solution is given by

$$
f(\eta)=f_{0}(\eta, \tau)+\sum_{m=1}^{\infty} f_{m}(\eta, \tau)
$$

where

$$
f_{m}(\eta, \tau)=\left.\frac{1}{m !} \frac{\partial^{m} \phi(\eta, \tau ; q)}{\partial q^{m}}\right|_{q=0}
$$

and $f_{m}$ is governed by $m^{\text {th }}$ order deformation equation 


$$
\mathcal{L}\left[f_{m}(\eta, \tau)-\chi_{m} f_{m-1}(\eta, \tau)\right]=c_{0} \mathcal{D}_{m-1}\{\mathcal{N}[\phi(\eta, \tau ; q)]\}
$$

where

$$
\chi_{m}= \begin{cases}0, & m \leq 1 \\ 1, & m>1\end{cases}
$$

with the initial and boundary conditions

$$
\begin{aligned}
& f_{m}(\eta, 0)=0, \\
& f_{m}(0, \tau)=\operatorname{Sin} \tau \text { or } \operatorname{Cos} \tau, \\
& f_{m}(\infty, \tau)=0 .
\end{aligned}
$$

Let $f_{m} *(\eta, \tau)$ denote the special solution of Eq.(3.9) with boundary conditions (3.10b) and (3.10c) and $\mathcal{L}^{-1}$ is inverse of the linear operator $\mathcal{L}, f_{m} *$ which is given by

$$
f_{m}^{*}(\eta, \tau)=\sum_{k=0}^{m} \alpha_{m-k} f_{k}(\eta, \tau)+c_{0} \sum_{k=0}^{m} \beta_{k} \mathcal{L}^{-1}\left[\mathcal{D}_{m-l}\{\mathcal{N}[\phi(\eta, \tau ; q)]\}\right]
$$

so that

$$
f_{m}^{*}(\eta, \tau)=\chi_{m} f_{m-1}(\eta, \tau)+c_{0} \mathcal{L}^{-1}\left[\mathcal{D}_{m-1}\{\mathcal{N}[\phi(\eta, \tau ; q)]\}\right]
$$

Homotopy series solutions for both sine and cosine oscillations are given by

$$
f(\eta)=f_{0}(\eta, \tau)+\sum_{m=1}^{\infty} f_{m}(\eta, \tau)=e^{-\eta} \operatorname{Sin} \tau+\sum_{m=1}^{\infty}\left\{f_{m}^{*}(\eta, \tau)+\left(\operatorname{Sin} \tau-f_{m}^{*}(0, \tau)\right) e^{-\eta}\right\}
$$

and

$$
f(\eta)=f_{0}(\eta, \tau)+\sum_{m=1}^{\infty} f_{m}(\eta, \tau)=e^{-\eta} \operatorname{Cos} \tau+\sum_{m=1}^{\infty}\left\{f_{m}^{*}(\eta, \tau)+\left(\operatorname{Cos} \tau-f_{m}^{*}(0, \tau)\right) e^{-\eta}\right\}
$$

After obtaining the velocity, the corresponding wall shear stresses can be obtained by means of the following relation

$$
\tau_{w}(\tau)= \begin{cases}\left.\eta_{0} \dot{\gamma}_{0} \frac{\partial f}{\partial \eta}\right|_{\eta \rightarrow 0} ; & \frac{\partial f}{\partial \eta} \leq 1 \\ \left.\eta_{0} \dot{\gamma}_{0}\left(\frac{\partial f}{\partial \eta}\right)^{n}\right|_{\eta \rightarrow 0} ; & \frac{\partial f}{\partial \eta} \geq 1\end{cases}
$$

Fetecau et al. [25] has discussed wall shear stress for $n=1$.

\section{Results and discussion}

After introducing similarity transformation (2.12), the governing Eq.(2.10) with initial condition (2.11a) and boundary conditions (2.11b) and (2.11c) is reduced to non dimensionalised partial differential 
Eqs (2.14) and (2.15) with initial and boundary conditions given in Eqs (2.16) and (2.17). Equation (2.14) is the case of a Newtonian fluid which is studied in [40] and has already been discussed. It is worth mentioning that for $n=1$, Eq.(2.14) and Eq.(2.15) are same. The nonlinear partial differential Eq.(2.15) with boundary conditions in Eqs (2.16) and (2.17) is solved via HAM, a semi analytic method. In this section, different values of the power index $n$ are considered to investigate the behaviour of flow of Spriggs fluid for second problem of Stokes'. It has been observed from the study that the non-Newtonian Spriggs fluid flows also acquire steady state like Newtonian fluid flows but it takes less time than for Newtonian flows to reach steady state.

\subsection{For $n=0.5$}

The simplified form of Eq.(2.15), is

$$
\frac{\partial f}{\partial \tau}=\frac{1}{2}\left(\frac{\partial f}{\partial \eta}\right)^{-0.5} \frac{\partial^{2} f}{\partial \eta^{2}}
$$

or

$$
\left(\frac{\partial f}{\partial \tau}\right)^{2}-4 \frac{\partial f}{\partial \eta}\left(\frac{\partial^{2} f}{\partial \eta^{2}}\right)^{2}=0
$$

\subsubsection{CASE (a): Subject to initial and boundary condition}

$$
f(\eta, 0)=0, \quad f(0, \tau)=\operatorname{Sin} \tau \quad \text { and } \quad f(\infty, \tau)=0 .
$$

In the HAM solution process, the convergence control parameter plays a very important role for giving convergent series solution. So to find the range of convergence control parameter $c_{0}$, we plot a graph between $f^{\prime}(0,0)$ and $c_{0}$ given in Fig. 3 for $2^{\text {nd }}, 3^{\text {rd }}$ and $4^{\text {th }}$ order of approximations. The series solution Eq.(3.13) for Eq.(4.1) will converge effectively in the range $-0.2 \leq c_{0} \leq 0.2$ for second, third and fourth approximations. For fourth order approximation, the range of $c_{0}$ is $-0.25 \leq c_{0} \leq 0.5$. Further, Fig.4 and Fig. 5 show the impact of $c_{0}$ over velocity $f$ along the tangential coordinate $\eta$ for different $c_{0}$ at $\tau=1.57$ and $\tau=4.71$, respectively. $c_{0}$. From both the figures, it is observed that as $c_{0}$ increases the velocity profile decreases more rapidly and it reveals that steadiness of the velocity profile can be adjusted and varied by using the convergence control parameter. The forth order approximation of the velocity profile $f$ is shown in Fig. 6 for $c_{0}=0.2$. It shows that the velocity profile for $\tau=8$ is higher and $\tau=5$ is lower among other values of $\tau$. The steadiness comes faster for $\tau=3$.

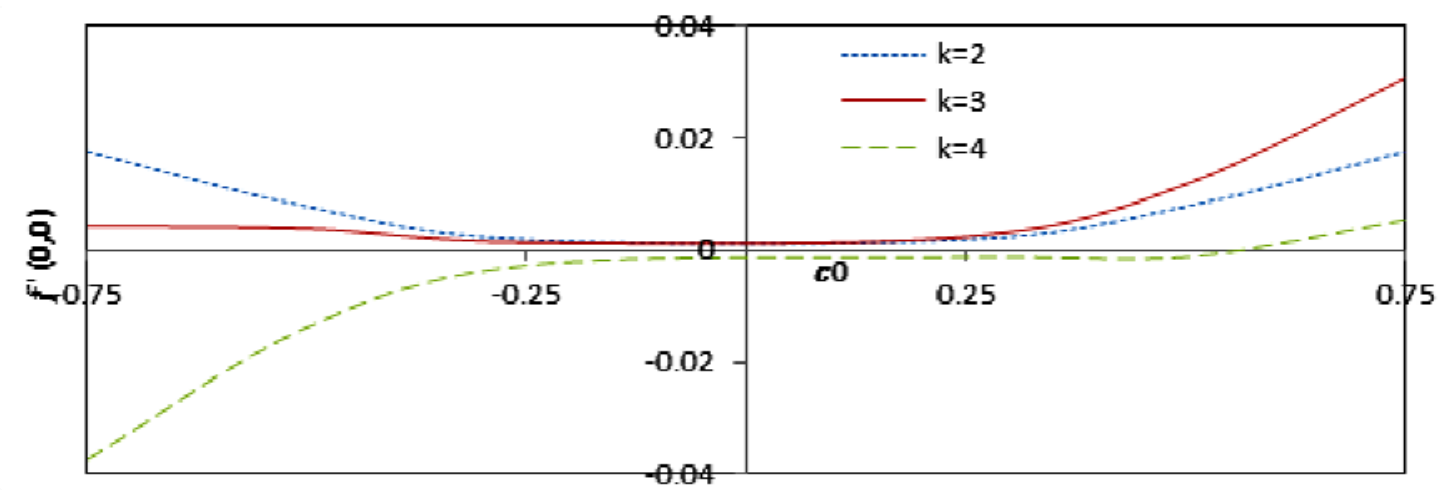

Fig.3. Variation of $f^{\prime}(0,0)$ and $c_{0}$ at $2^{\text {nd }}, 3^{\text {rd }}$ and $4^{\text {th }}$ order of approximation. 


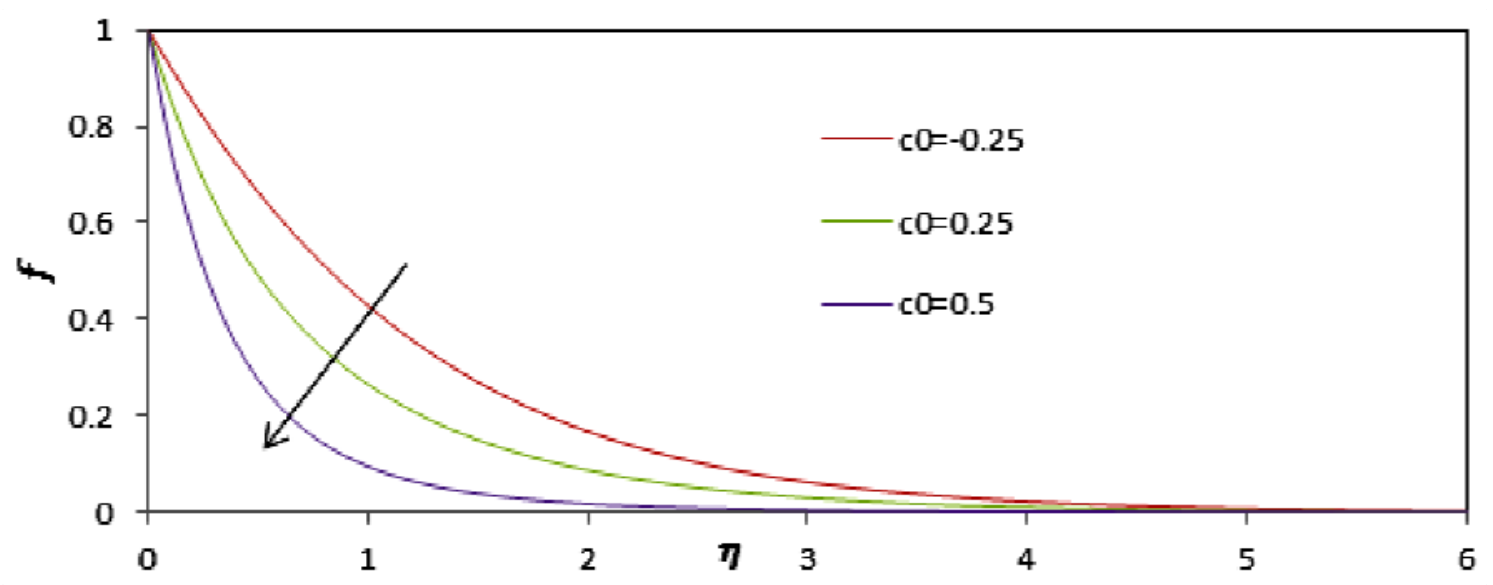

Fig.4. Effect of $c_{0}$ on velocity $f$ at $\tau=1.57$.

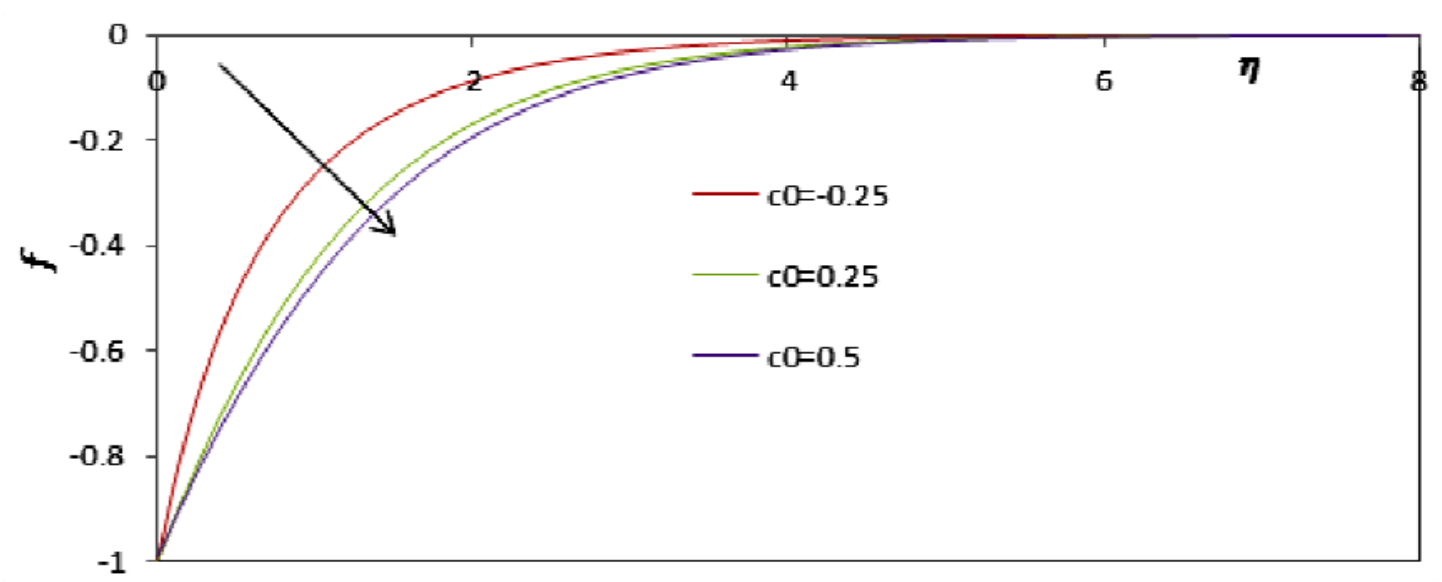

Fig.5. Effect of $c_{0}$ on velocity $f$ at $\tau=4.71$.

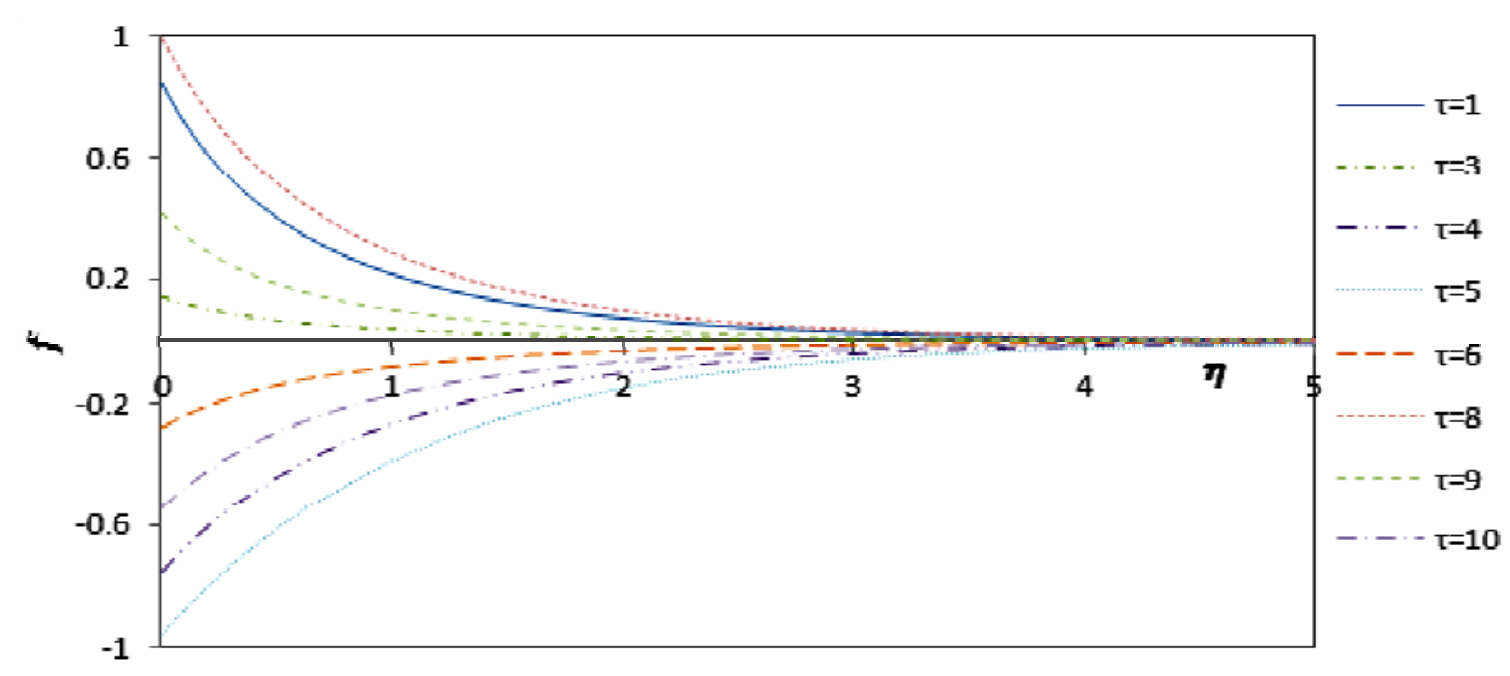

Fig.6. Influence of time $\tau$ on velocity $f$ for $c_{0}=0.2$ in the case of sine oscillation. 


\subsubsection{CASE (b): Subject to initial and boundary conditions}

$$
f(\eta, 0)=0, \quad f(0, \tau)=\operatorname{Cos} \tau \quad \text { and } \quad f(\infty, \tau)=0 .
$$

Variation of $f^{\prime}(0,0)$ versus $c_{0}$ is presented in Fig.7, for $2^{\text {nd }}, 3^{\text {rd }}$ and $4^{\text {th }}$ order of approximations to show the range of $c_{0}$. It illustrates that the series solution (3.14) of Eq.(4.1) in this case converges for $-0.75 \leq c_{0}<-0.1$. The fourth order approximation shows the range $-0.9<c_{0}<-0.05$.

\section{Effect of convergence control parameter $c_{0}$ over velocity}

Figures 8 and 9 show the variation of $f$ with respect to $\eta$ for different $c_{0}$ at $\tau=3.14$ and $\tau=6.28$, respectively. Also, it is observed that the velocity reduces with an increase in $c_{0}$. The values of $c_{0}$ are taken from the range of $c_{0}$. Figure10 shows variation of the velocity profile at various values of dimensionless time $\tau$.

It is found that initialy, i.e., at $\tau=0$ the highest velocity observed while at $\tau=2$ the the velocity is lowest, other velociti profiles are lie between them. It is seen that the steadiness comes faster for $\tau=3$ and at $\eta=0$ the velocity is lowest for $\tau=3$.

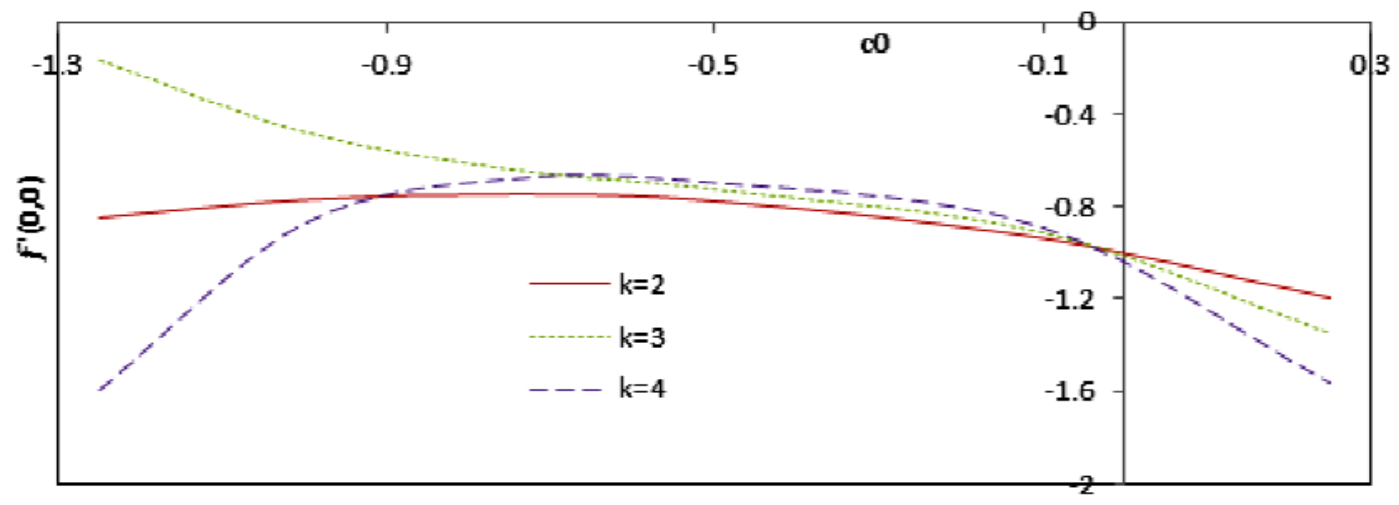

Fig.7. Variation of $f^{\prime}(0,0)$ and $c_{0}$ at $2^{\text {nd }}, 3^{\text {rd }}$ and $4^{\text {th }}$ order of approximation.

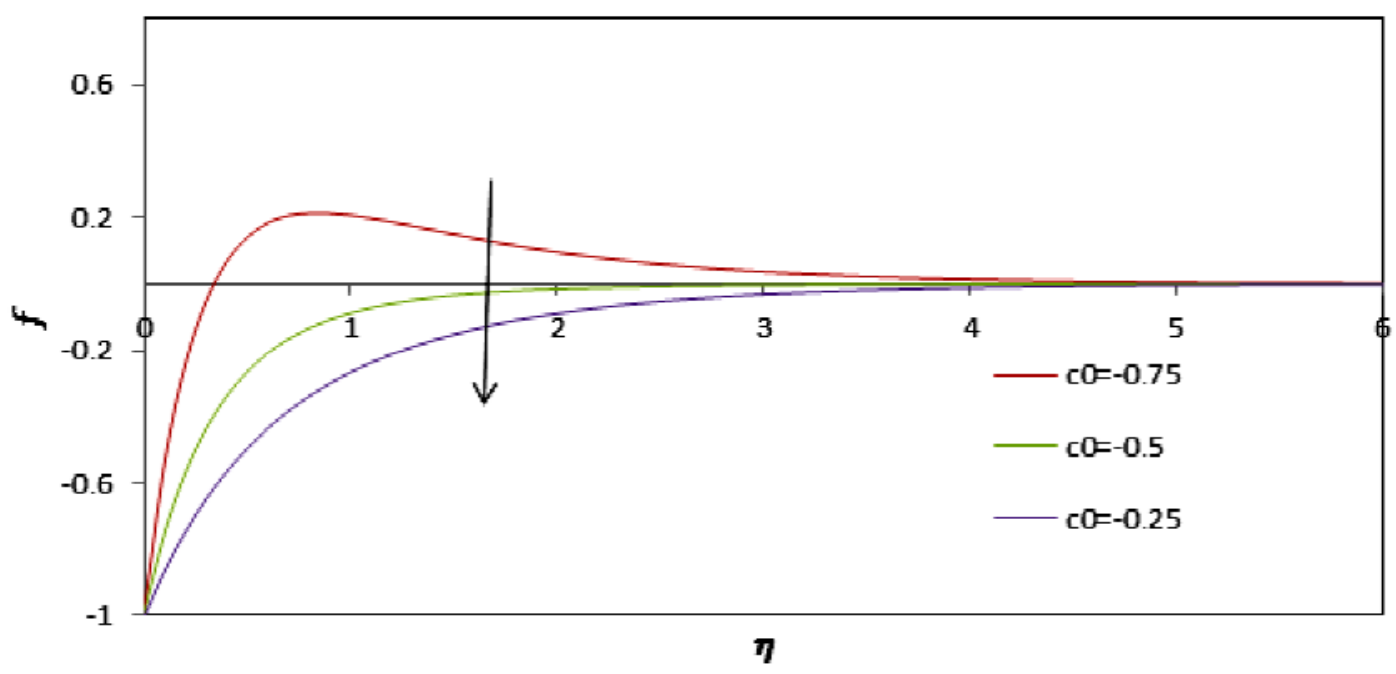

Fig.8. Effect of $c_{0}$ on velocity $f$ for $4^{\text {th }}$ order of approximation at $\tau=3.14$. 


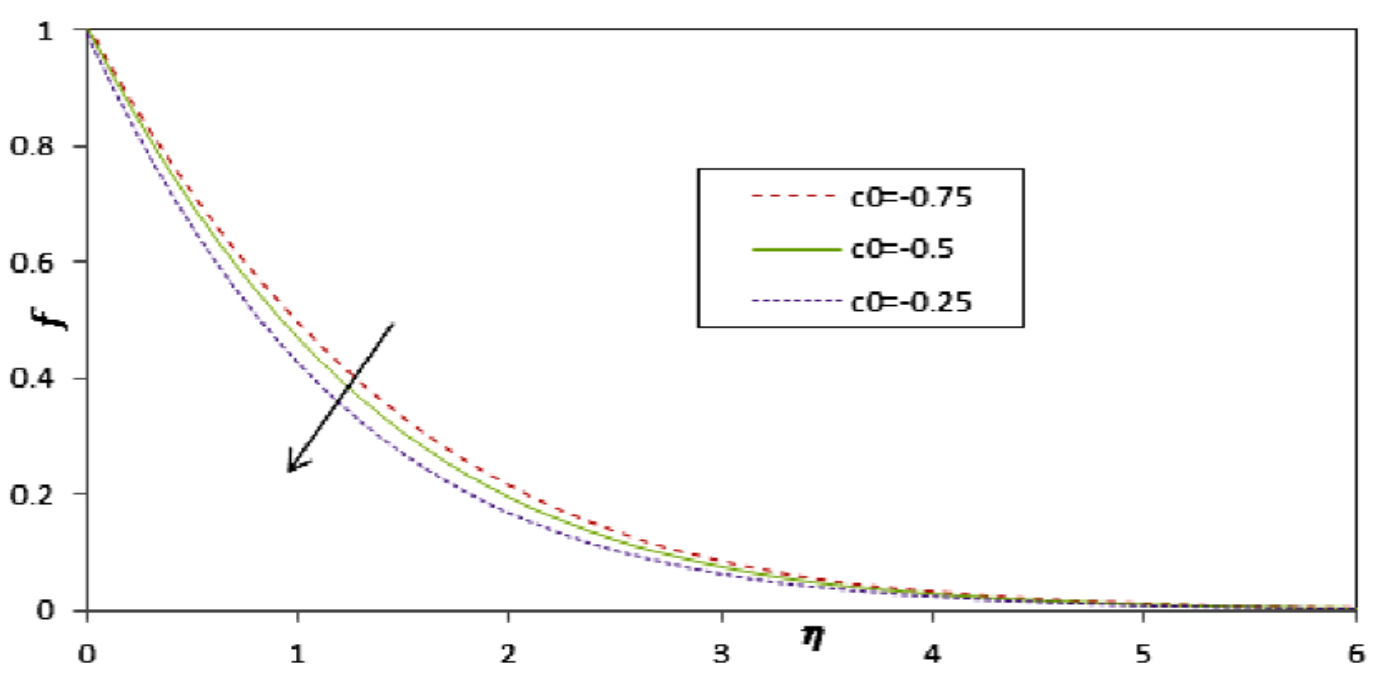

Fig.9. Effect of $c_{0}$ on velocity $f$ for $4^{\text {th }}$ order of approximation at $\tau=6.28$.

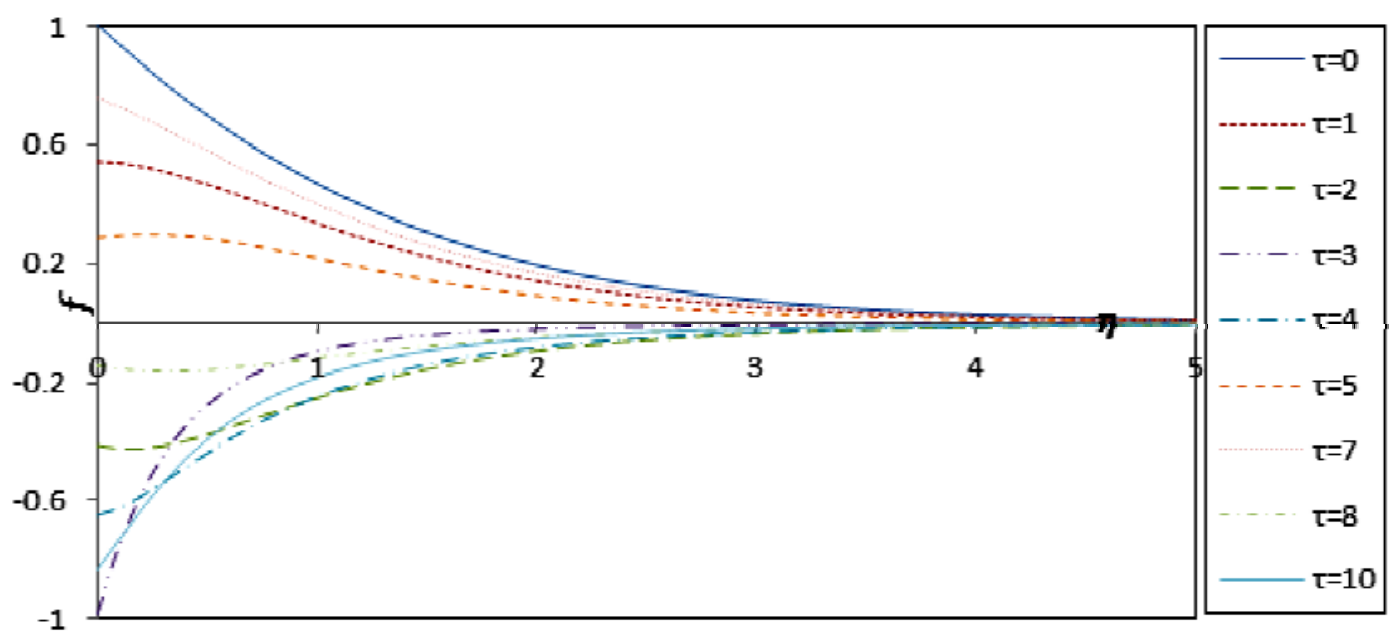

Fig.10. Influence of time $\tau$ on $4^{\text {th }}$ order approximation of velocity $f$ for $c_{0}=-0.25$ in case of cosine oscillation.

\subsubsection{Wall shear stress $\tau_{w}$ for $n=0.5$}

$$
\tau_{w}(\tau)=\left.\eta_{0} \dot{\gamma}_{0}\left(\frac{\partial f}{\partial \eta}\right)^{0.5}\right|_{\eta \rightarrow 0}
$$

Figure 11 shows the sine and cosine variation of $\tau_{w}$ over several time intervals. Here $\eta_{0} \dot{\gamma}_{0}=1$ and $c_{0}=-0.05$ is considered for sine and $c_{0}=-0.1$ is taken for cosine oscillations. 


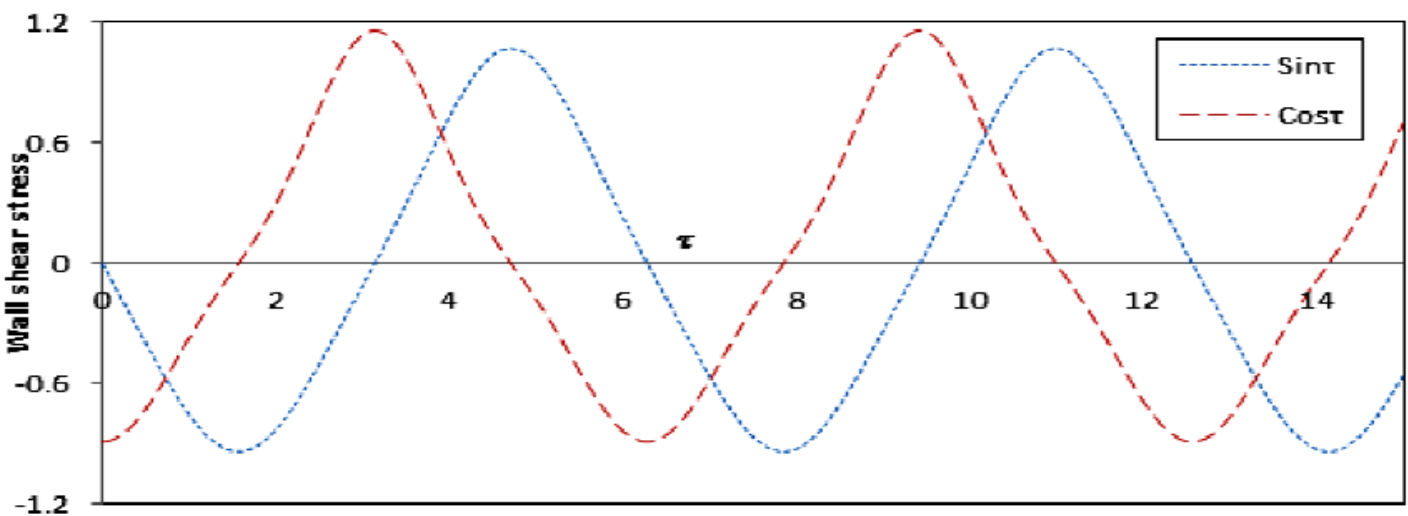

(a)

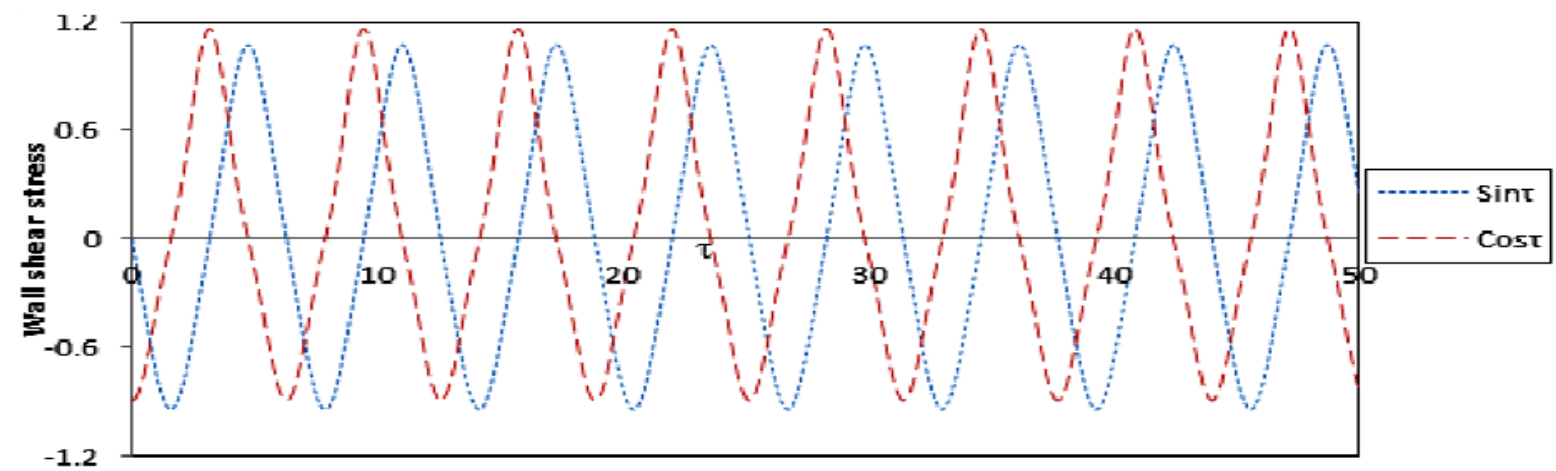

(b)

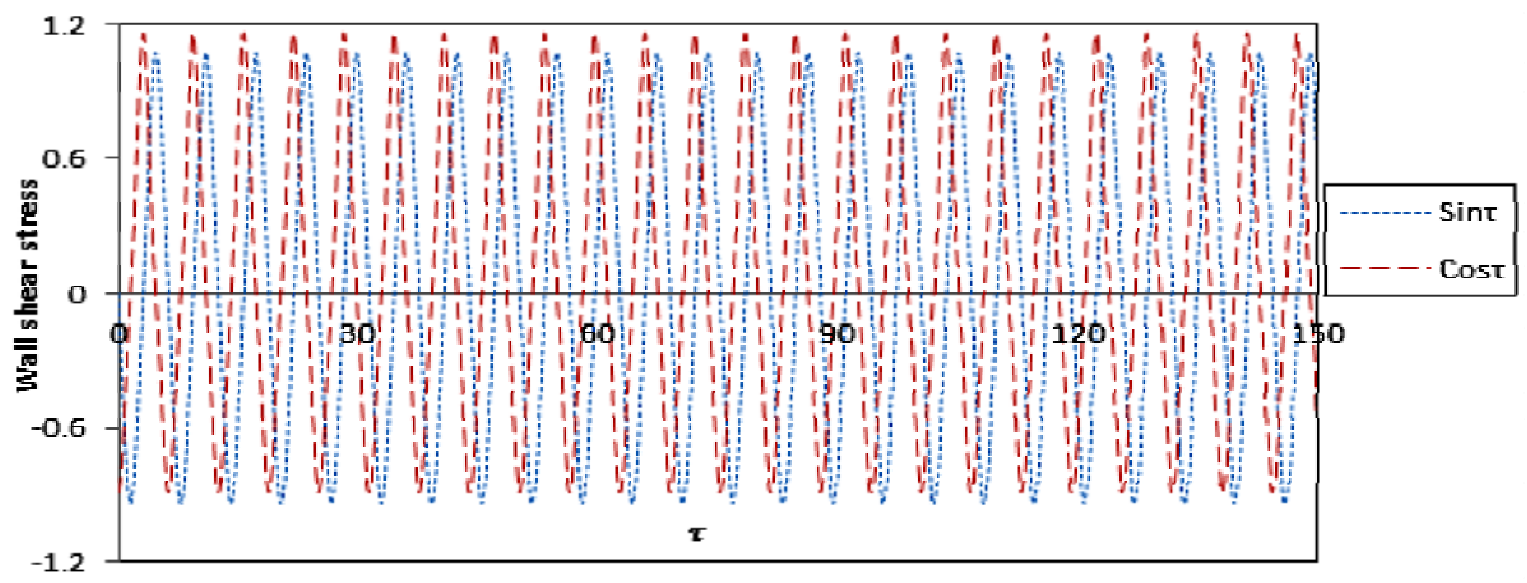

(c)

Fig.11. Variation of $\tau_{w}$ over time for sine and cosine oscillations for several time intervals (a) $\tau \in[0,15]$ (b) $\tau \in[0,50]$ (c) $\tau \in[0,150]$.

It is observed that as the value of $\eta_{0} \dot{\gamma}_{0}$ increases the amplitude of $\tau_{w}$ increases. While the behaviour of $\tau_{w}$ will a similar.

\subsection{For $n=0.75$}

The form of Eq.(2.15) will become 


$$
\frac{\partial f}{\partial \tau}=\frac{3}{4}\left(\frac{\partial f}{\partial \eta}\right)^{-1 / 4} \frac{\partial^{2} f}{\partial \eta^{2}},
$$

or

$$
\left(\frac{\partial^{2} f}{\partial \eta^{2}}\right)^{4}-\frac{256}{81} \frac{\partial f}{\partial \eta}\left(\frac{\partial f}{\partial \tau}\right)^{4}=0
$$

\subsubsection{CASE (a): For conditions}

$$
f(\eta, 0)=0, \quad f(0, \tau)=\operatorname{Sin} \tau \quad \text { and } \quad f(\infty, \tau)=0 .
$$

Variation of $f^{\prime}(0,0)$ versus $c_{0}$ is shown in Fig.12, for $3^{\text {rd }}, 4^{\text {th }}$ and $5^{\text {th }}$ order of approximations. Series solution (3.13) converges in the range $-0.25<c_{0}<0.25$ for the present case. The velocity profile $f$ for $c_{0}=-0.25$ is shown in Fig.13 and displays that the velocity profile for $\tau=8$ is higher and at $\tau=5$, the velocity profile is lower, other velocity profiles are in between them. The steadiness comes faster for $\tau=3$. It is clearly shown in Fig.13 that the term in initial condition is dominating, the velocity profile is approaching zero as the $\eta$ increases for the exponential term and velocity shows the harmonic (sine and cosine) behaviour as well.

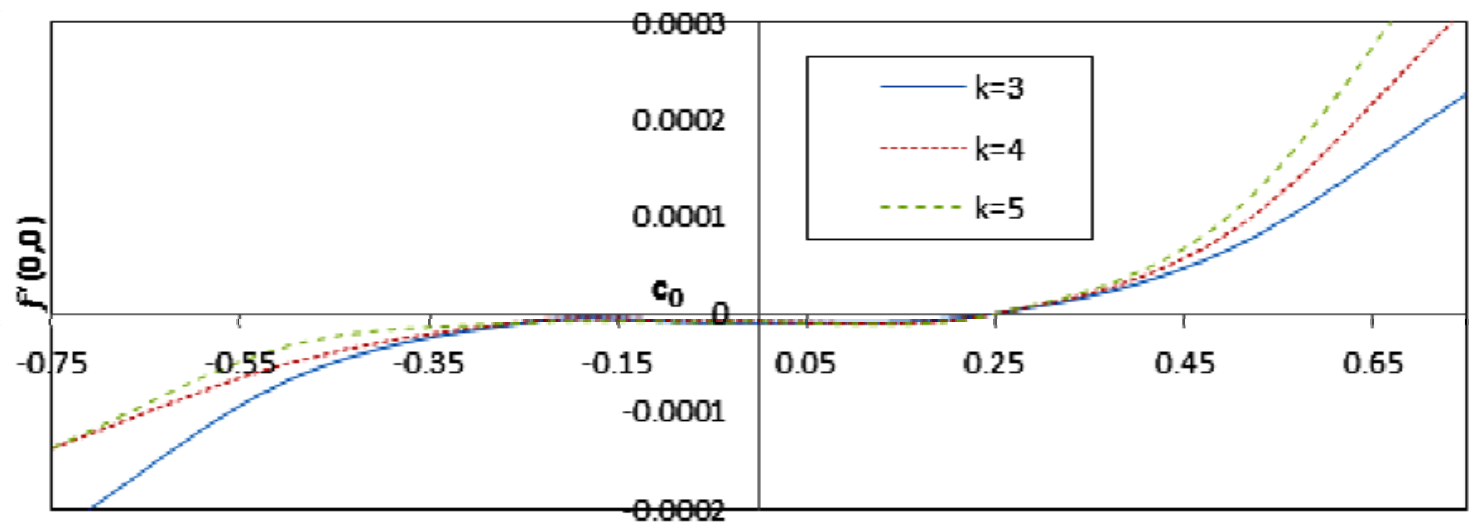

Fig.12. Variation of $f^{\prime}(0,0)$ vs $c_{0}$ at $3^{\text {rd }}, 4^{\text {th }}$ and $5^{\text {th }}$ order of approximation.

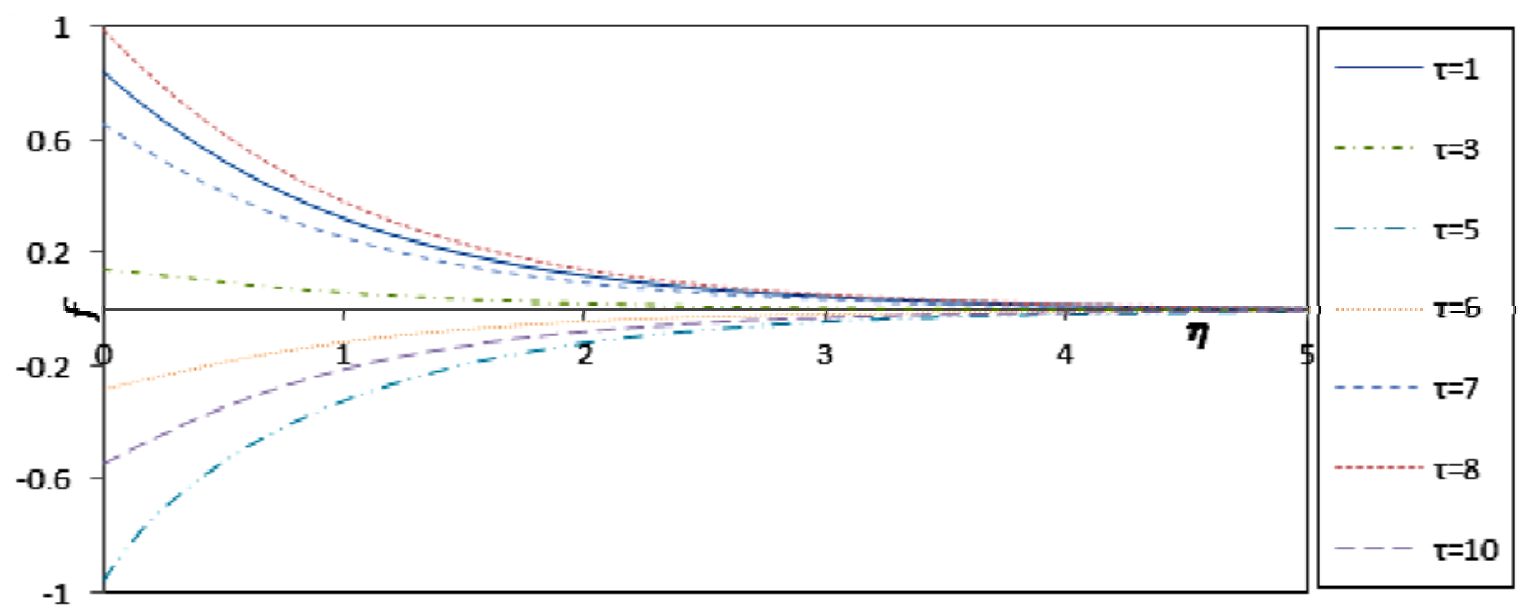

Fig.13. Influence of time $\tau$ on velocity $f$ at $c_{0}=-0.25$ for sine oscillation. 


\subsubsection{CASE (b): For conditions}

$$
f(\eta, 0)=0, \quad f(0, \tau)=\operatorname{Cos} \tau \quad \text { and } \quad f(\infty, \tau)=0 .
$$

Variations of $f^{\prime}(0,0)$ versus $c_{0}$ are presented in Fig. 14 for $2^{\text {nd }}, 3^{\text {rd }}$ and $4^{\text {th }}$ order of approximations. The series solution (3.14) in this case converges effectively in the range $-0.45 \leq c_{0}<-0.1$. The velocity profile $f$ for various $\tau$ at $c_{0}=-0.25$ is shown in Fig.15. It is observed that the velocity profile for $\tau=0$ is higher and at $\tau=3$, the velocity profile is lower. The steadiness comes faster for $\tau=8$.

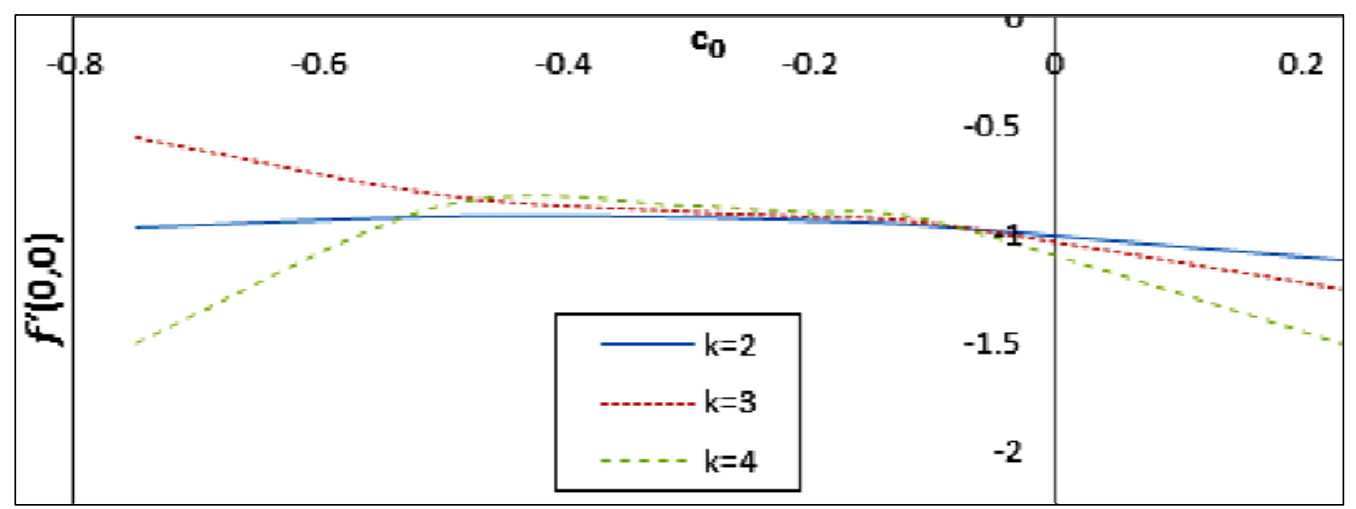

Fig.14. Variation of $f^{\prime}(0,0)$ and $c_{0}$ at $2^{\text {nd }}, 3^{\text {rd }}$ and $4^{\text {th }}$ order of approximation.

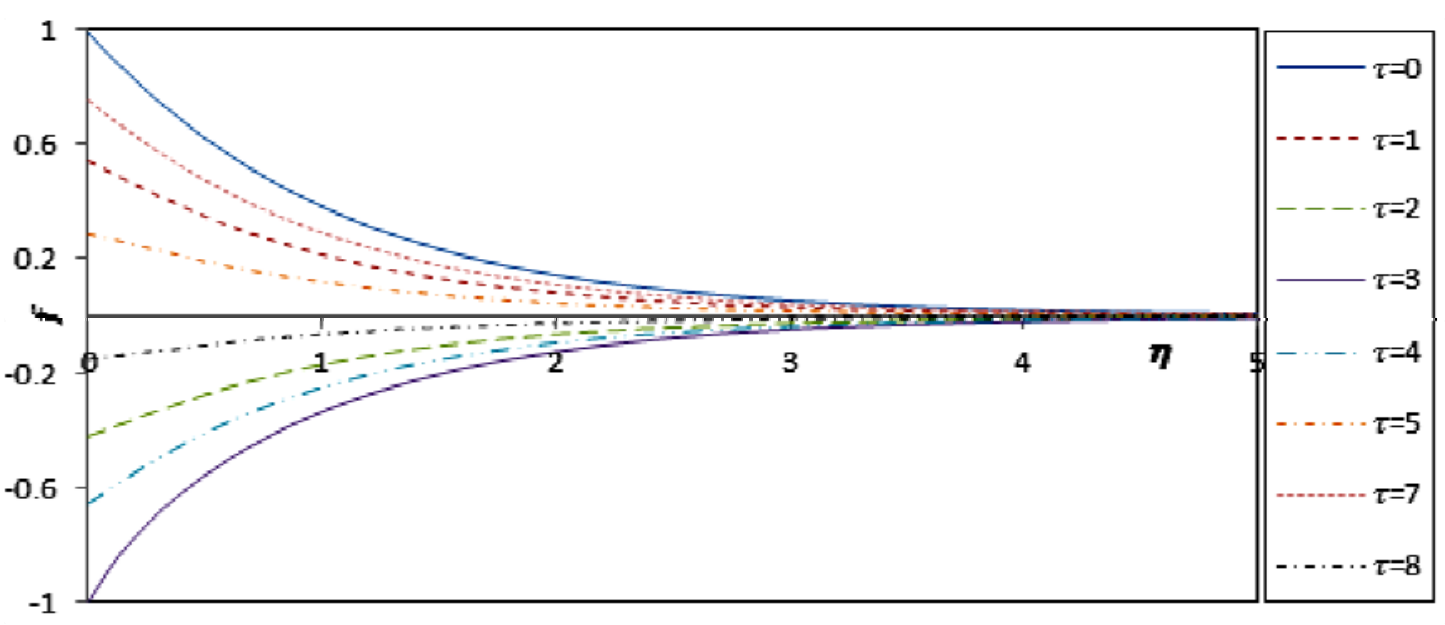

Fig.15. Influence of time $\tau$ on $f$ at $c_{0}=-0.25$ for cosine oscillation.

\subsubsection{Wall shear stress for $n=0.75$}

The behaviour of wall shear stress $\tau_{w}$ for both oscillations is shown in Fig.16 at different time intervals. The wall shear stress $\tau_{w}$ for $n=0.75$ is given by

$$
\tau_{w}(\tau)=\left.\eta_{0} \dot{\gamma}_{0}\left(\frac{\partial f}{\partial \eta}\right)^{0.75}\right|_{\eta \rightarrow 0}
$$


where $\eta_{0} \dot{\gamma}_{0}=1$ and $c_{0}=-0.1$ is considered for both sine and cosine oscillations.

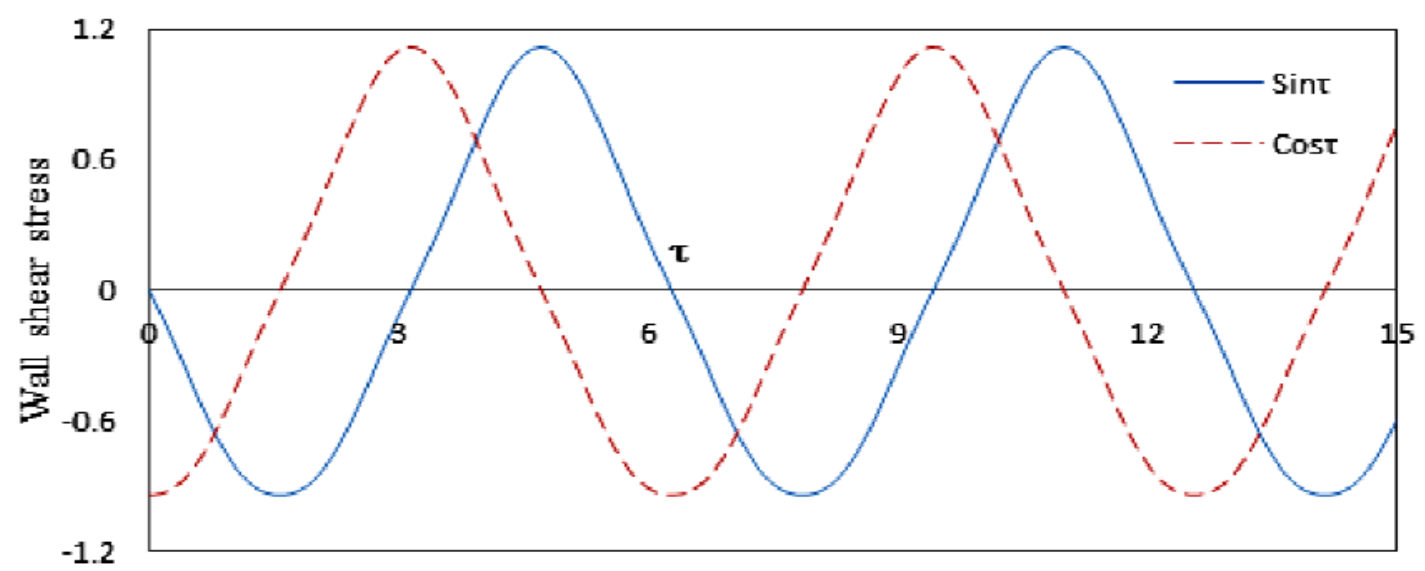

(a)

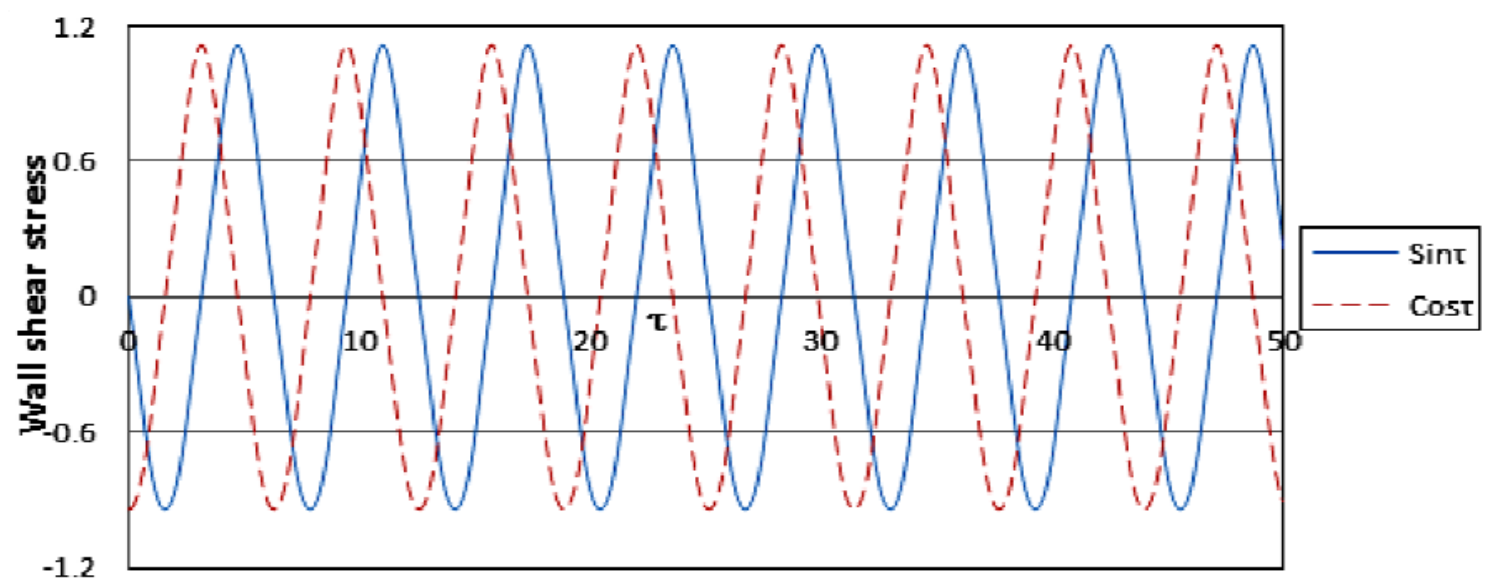

(b)

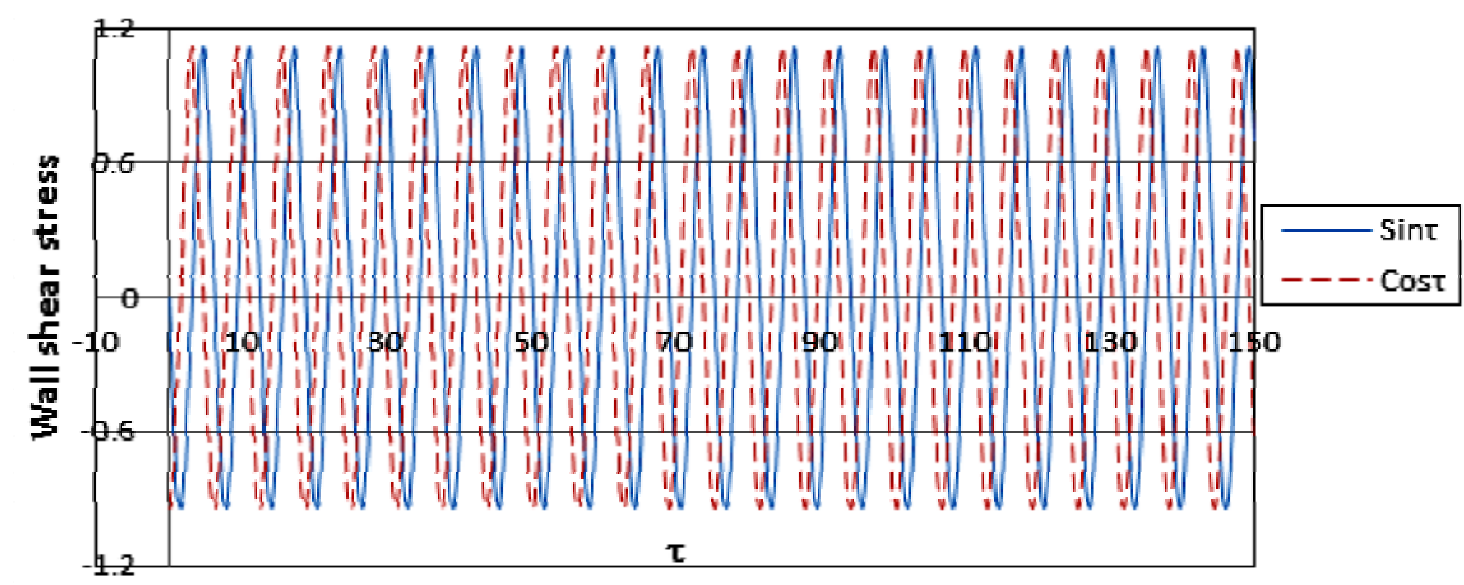

(c)

Fig.16. Variation of $\tau_{w}$ over time for sine and cosine oscillations for several time intervals (a) $\tau \in[0,15]$ (b) $\tau \in[0,50]$ (c) $\tau \in[0,150]$. 


\subsubsection{The behaviour of shear thinning}

The velocity profile for $n=0.5$ and $n=0.75$ is shown in Figs 17 and 18 when the boundary conditions are $f(0, \tau)=\operatorname{Sin} \tau$ and $f(0, \tau)=\operatorname{Cos} \tau$, respectively. Here, $c_{0}=-0.25$. The solid line shows the velocity profile for a Newtonian fluid. The dot line and dash line represent the velocity profile for shear thinning fluid behaviour i.e., for $n=0.5$ and $n=0.75$, respectively. Figure 17 demonstrates that the steadiness for $n=0.75$ comes early with respect to $n=0.5$ at $\tau=1.57$ and steadiness for $n=0.5$ comes earlier than $n=0.75$ at $\tau=4.71$. Note that there is no phase change for shear thinning flow while there is phase change in the Newtonian flow. Figure 18 indicates that the steadiness for $n=0.75$ is delayed than for $n=0.5$.

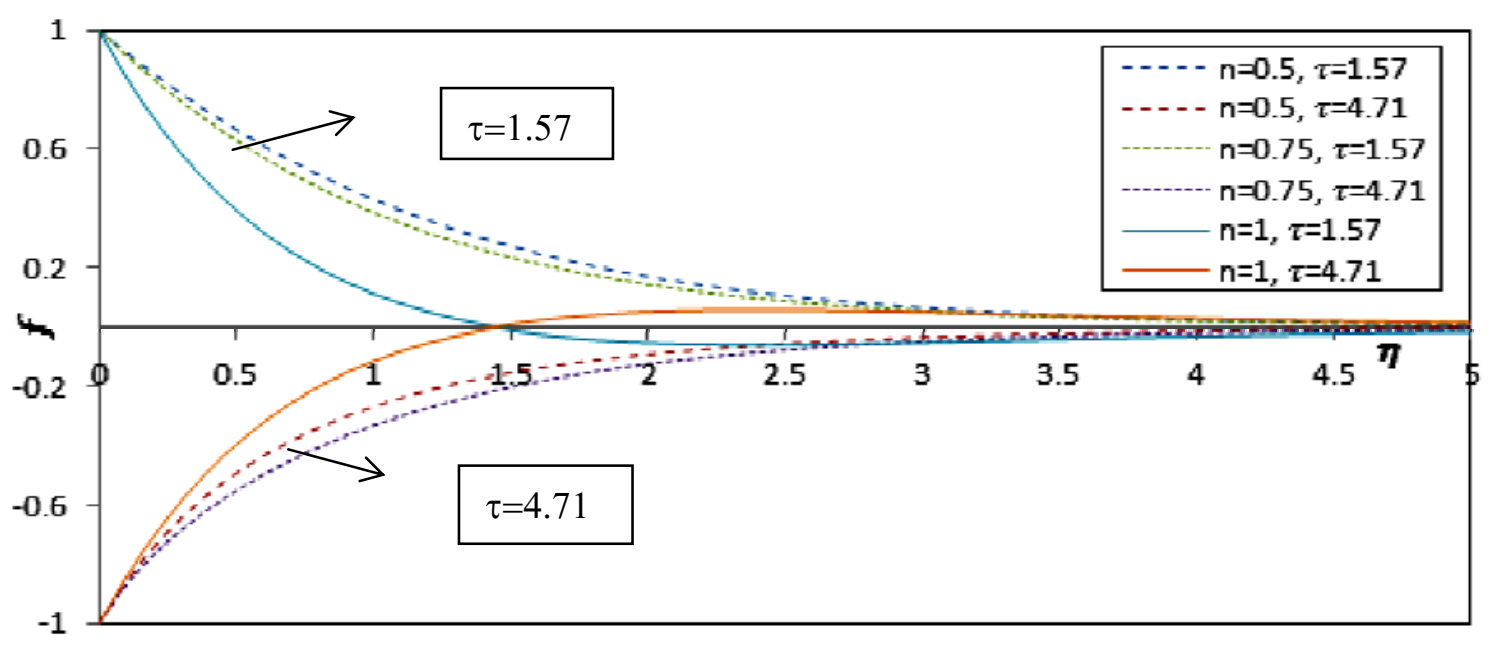

Fig.17. Velocity profile for different $n$ and $\tau$ for sine oscillation.

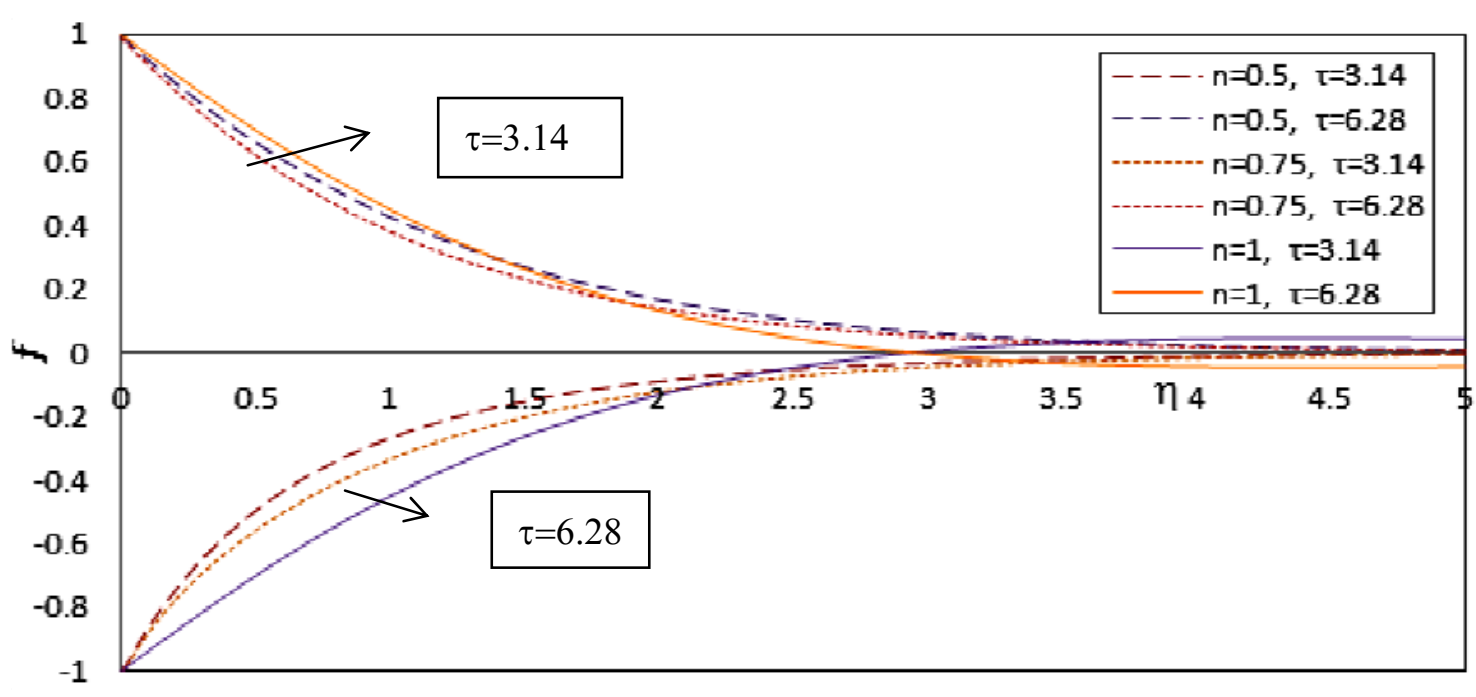

Fig.18. Velocity profile for different $n$ and $\tau$ for cosine oscillation.

\subsection{For $n=1.5$}

From Eq.(2.15) we have 


$$
\frac{\partial f}{\partial \tau}=\frac{3}{2}\left(\frac{\partial f}{\partial \eta}\right)^{1 / 2} \frac{\partial^{2} f}{\partial \eta^{2}}
$$

or

$$
\left(\frac{\partial f}{\partial \tau}\right)^{2}-\frac{9}{4} \frac{\partial f}{\partial \eta}\left(\frac{\partial^{2} f}{\partial \eta^{2}}\right)^{2}=0
$$

\subsubsection{CASE (a): For the initial and boundary condition}

$$
f(\eta, 0)=0, \quad f(0, \tau)=\operatorname{Sin} \tau \quad \text { and } \quad f(\infty, \tau)=0 .
$$

The velocity profile $f$ with sixth order approximation for $c_{0}=-0.25$ is shown in Fig.19.

It shows that the highest velovcity value is for $\tau=8$ and the lowest velovcity value is for $\tau=5$. The steadiness comes faster for $\tau=6$.

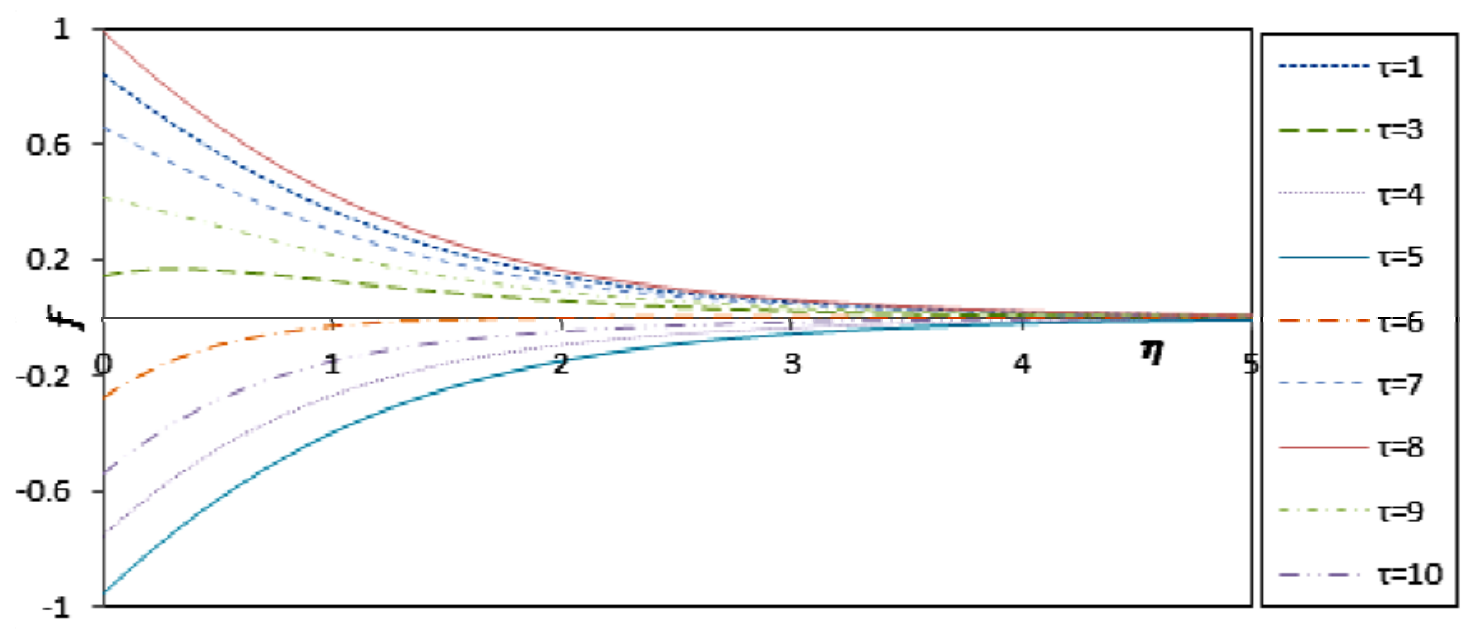

Fig.19. Influence of time $\tau$ on $6^{\text {th }}$ order approximation of velocity $f$ for sine oscillation and $c_{0}=-0.25$.

\subsubsection{CASE (b): For the initial and boundary condition}

$$
f(\eta, 0)=0, \quad f(0, \tau)=\operatorname{Cos} \tau \quad \text { and } \quad f(\infty, \tau)=0 .
$$

Variation of $f^{\prime}(0,0)$ and $c_{0}$ is given in Fig.20, for $3^{\text {rd }}, 4^{\text {th }}$ and $5^{\text {th }}$ order of approximations. It indicates that the solution (3.14) converges better in the range $-0.4<c_{0}<0.1$ in the present case. The variation of velocity $f$ at various values of $\tau$ is shown in Fig. 21 for fifth order of approximation and $c_{0}=-0.25$. For $\tau=0$ is the highest velocity whereas for $\tau=3$ the velocity is lowest and steadiness comes earlier for $\tau=2$. 


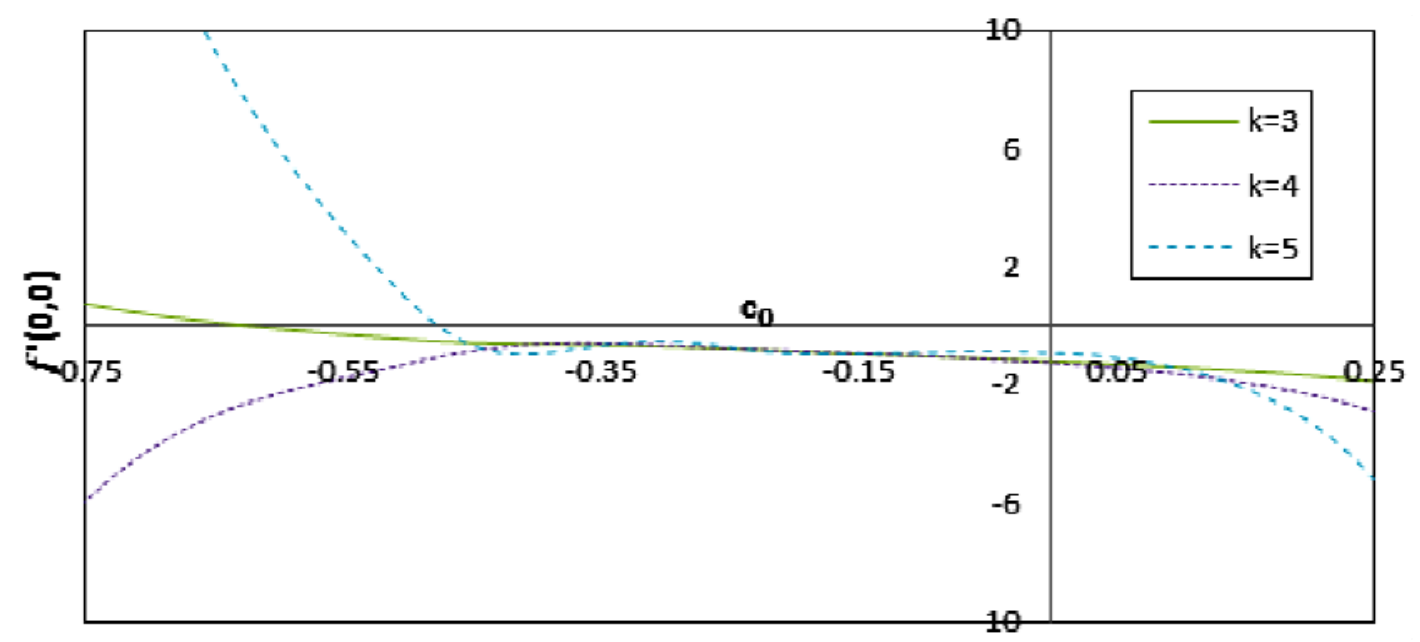

Fig.20. Variation of $f^{\prime}(0,0)$ vs $c_{0}$ at $3^{\text {rd }}, 4^{\text {th }}$ and $5^{\text {th }}$ order of approximation.

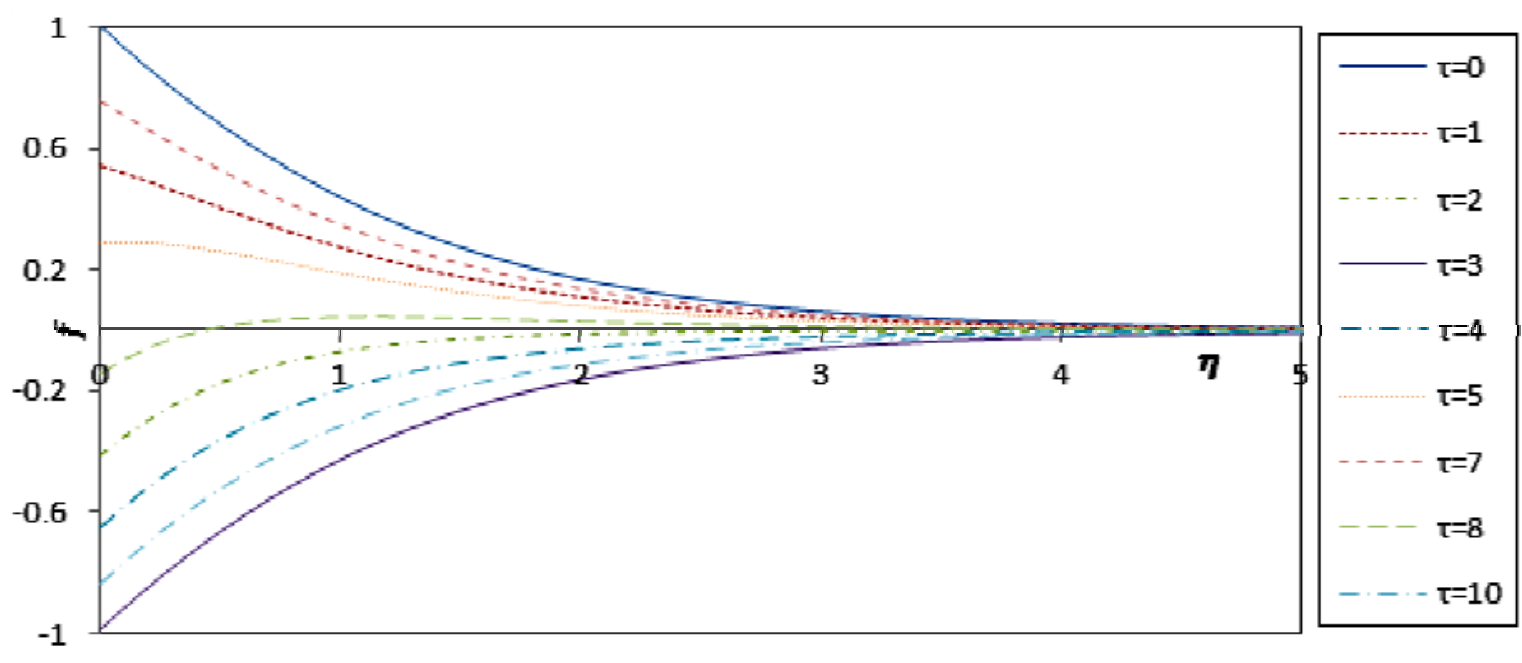

Fig.21. Influence of time $\tau$ over $5^{\text {th }}$ order approximation of velocity $f$ for $c_{0}=-0.25$ for cosine oscillation.

\subsubsection{Wall shear stress for $n=1.5$}

The graphs of the fifth order approximation of $\tau_{w}$, corresponding to the cosine and sine oscillations of the boundary are shown in Fig.22 at different time intervals for $c_{0}=-0.1$. Amplitudes for both oscillations are similar and have shift of phase which holds for all times.

$$
\tau_{w}(\tau)=\left.\eta_{0} \dot{\gamma}_{0}\left(\frac{\partial f}{\partial \eta}\right)^{1.5}\right|_{\eta \rightarrow 0}
$$

Here $\eta_{0} \dot{\gamma}_{0}=1$ is considered. 


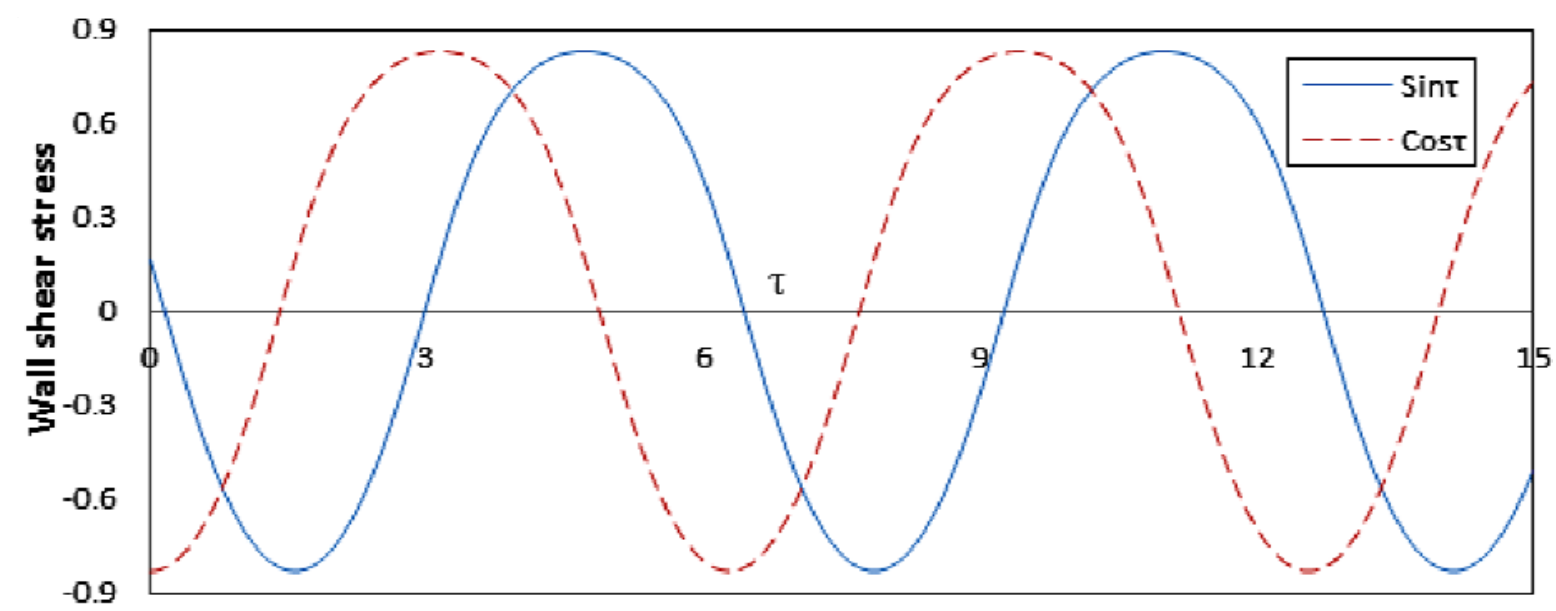

(a)

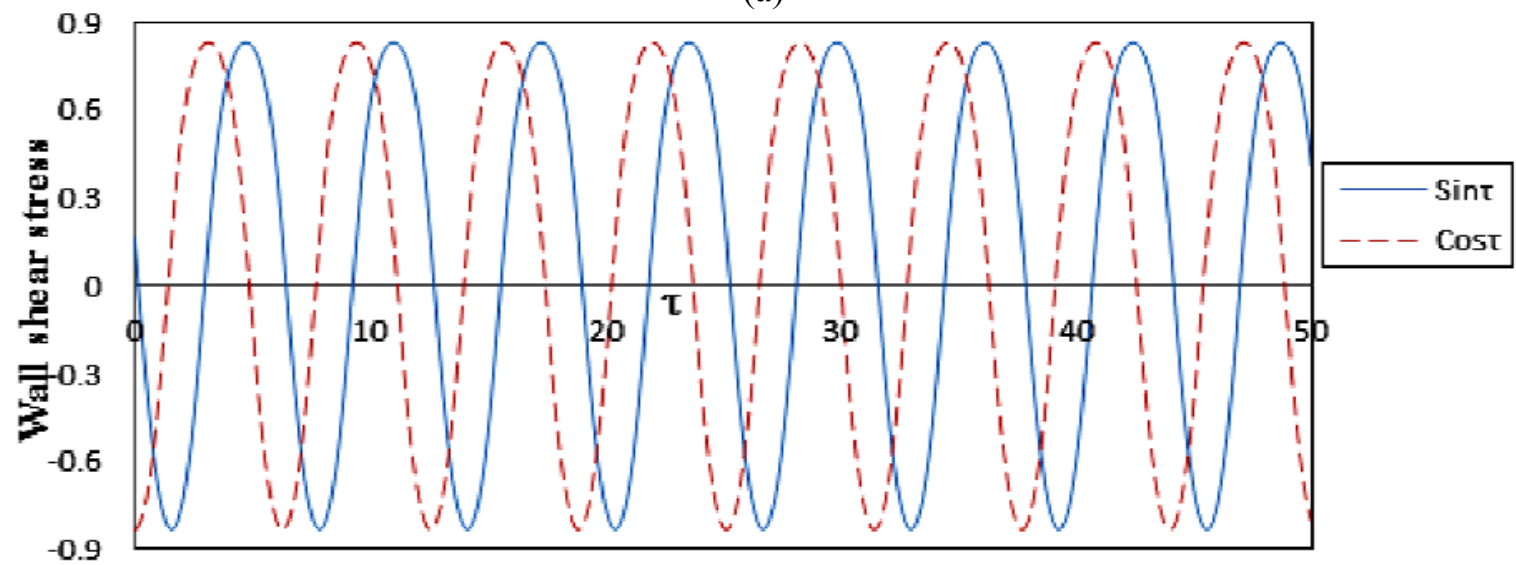

(b)

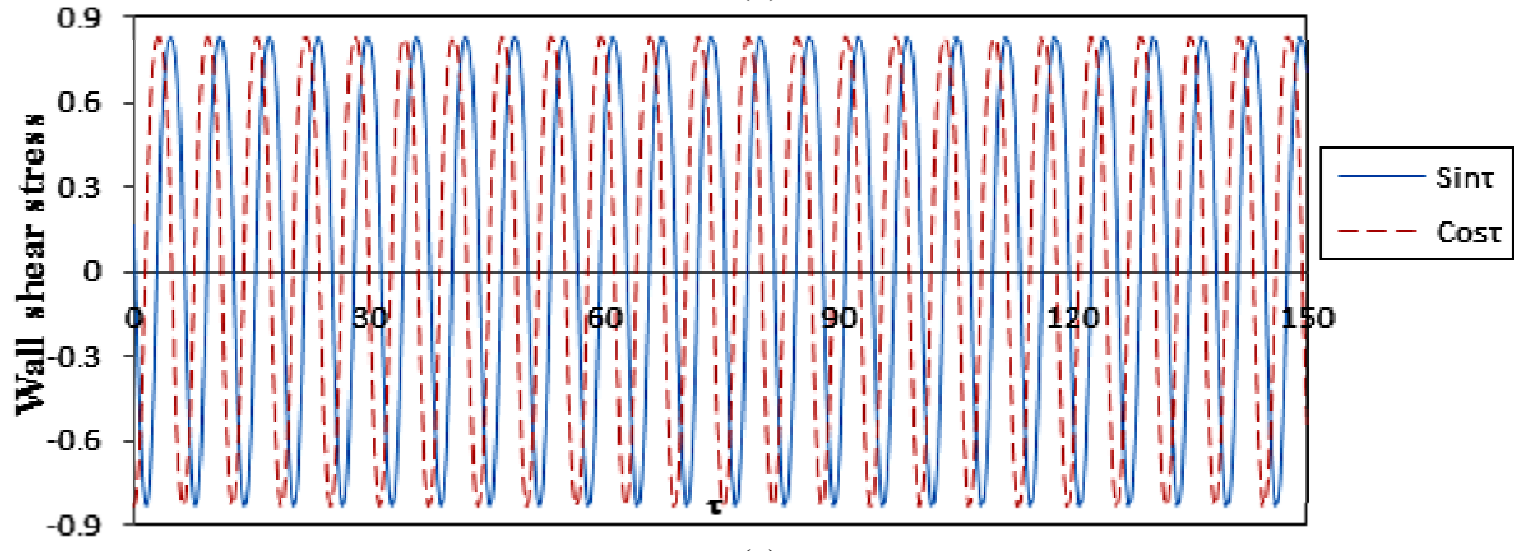

(c)

Fig.22. Variation of $\tau_{w}$ over time for sine and cosine oscillations for several time intervals $(\mathrm{a}) \tau \in[0,15]$ (b) $\tau \in[0,50]$ (c) $\tau \in[0,150]$.

\subsubsection{Behaviour of shear thickening}

The behaviour for $n=2$ and $n=3$ over velocity profile is given in Figs 23 and 24 for sine and cosine oscillations, respectively. Forth order approximation for the velocity at $\tau=1.57,3.14,4.71$ and 6.28 and $c_{0}=-0.25$ is shown in these figures. The solid line shows the velocity profile for a Newtonian fluid and 
dot and dash lines show the velocity profile for shear thickening fluid behaviour, i.e. for $n=2$ and $n=3$, respectively. The figure explains that there is no shift of phase for shear thinning and shear thickening fluids while there is phase shift for Newtonian fluids. The steadiness for $n=2$ comes early with respect to $n=3$.

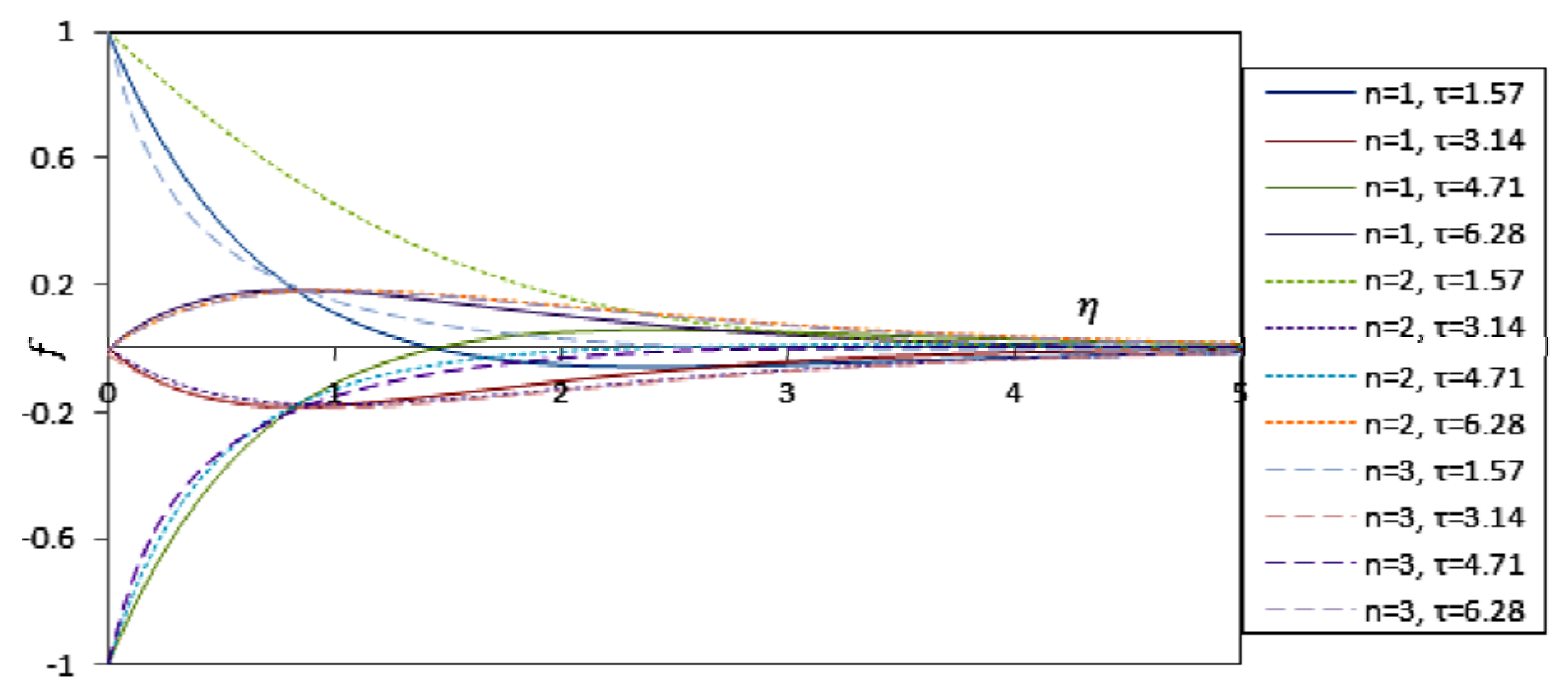

Fig.23. Velocity profile for different $n$ and $\tau$ for sine oscillation.
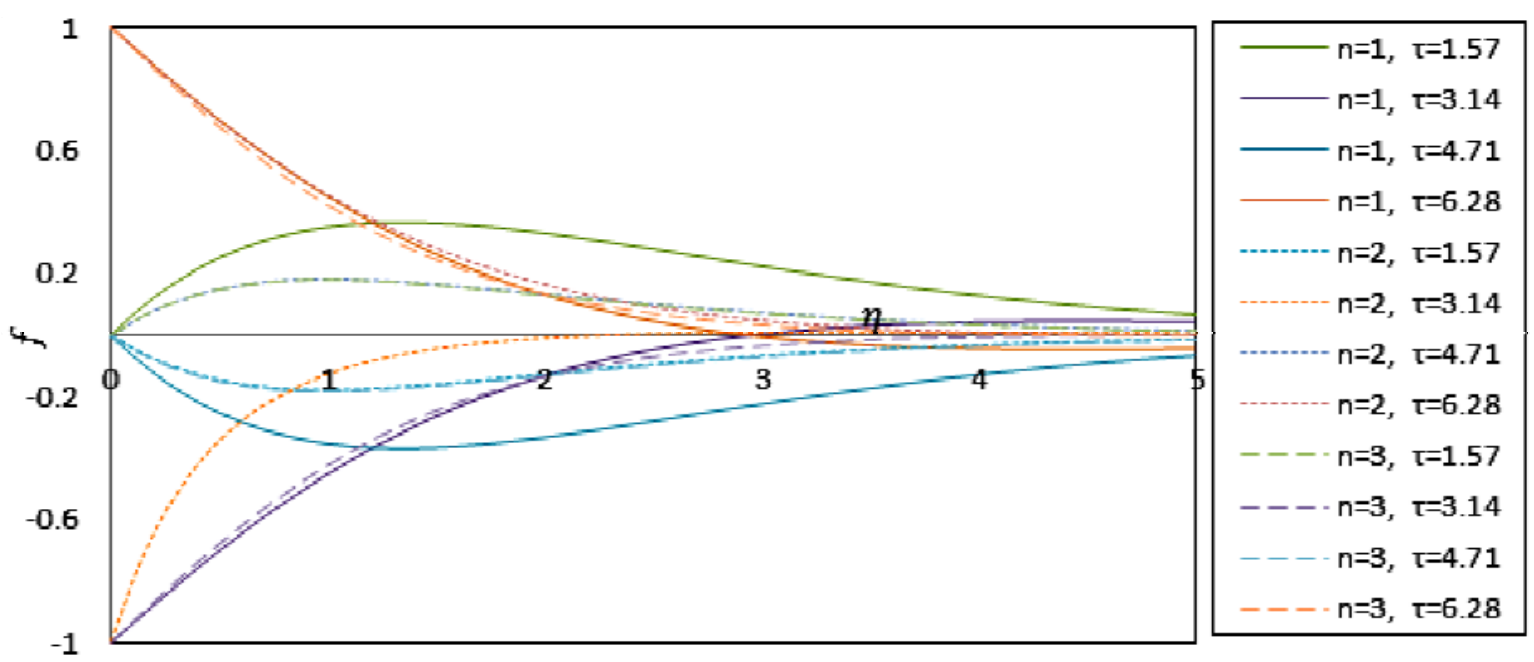

Fig.24. Velocity profile for different $n$ and $\tau$ for cosine oscillation.

\subsection{Influence of parameter $n$ over velocity profile for fixed $\tau$}

The fourth order approximation of the velocity profile for a fixed $\tau$ is shown for different $n$.

CASE (a): For principle boundary condition $f(0, \tau)=\operatorname{Sin} \tau$

Figure 25 illustrates the velocity at $\tau=1.57$. It shows that the steadiness of a Newtonian fluid comes for $\eta=8.5$ onwards, while for a shear thinning fluid $(n=0.5, n=0.75)$ the steadiness comes at $\eta=7.5$ and steadiness of a shear thickening fluid ( $n=1.5)$ came early at $\eta=5$. Figure 26 shows velocity at $\tau=4.71$ and denotes the same behaviour as it is shown in Fig.25. Also as the power index $n$ increases, the velocity profile decrease for a shear thinning fluid. As the viscosity of a shear thickening fluid increases after 
applying shear stress, so velocity will decays to zero earlier for a shear thickening fluid than the shear thinning fluid for which the viscosity decreases by applying shear stress. As a result, velocity of a shear thickening fluid decays to zero earlier than for the shear thinning fluid.

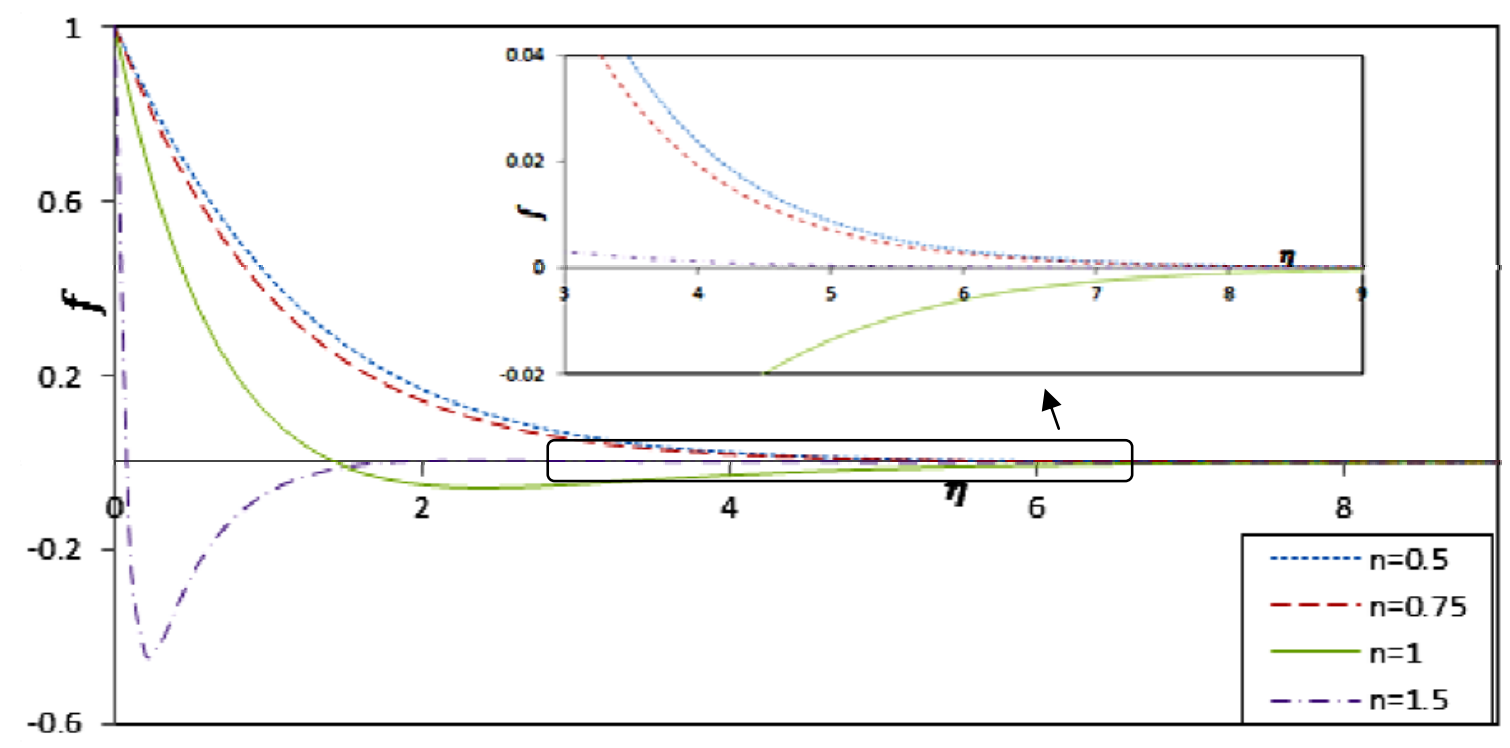

Fig.25. Effect of $n$ on velocity profile at $\tau=1.57$ for sine oscillation.

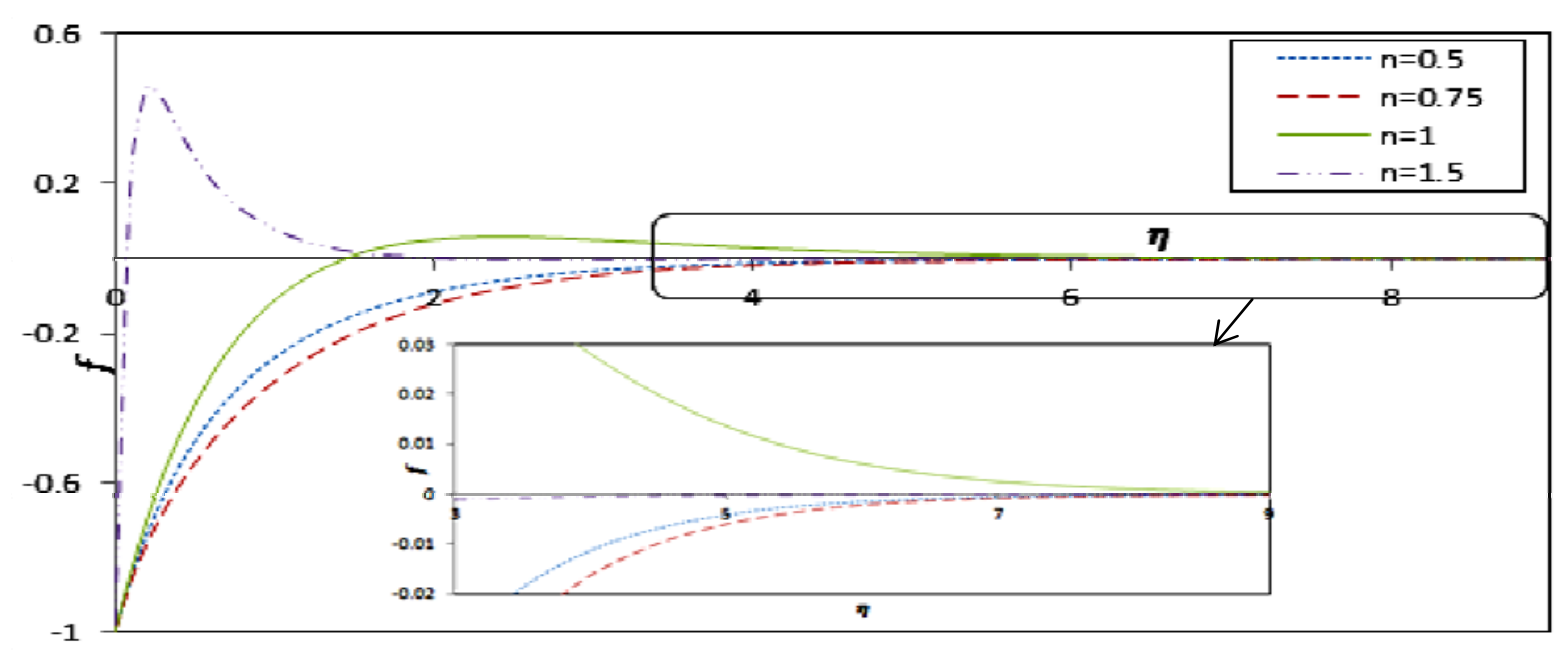

Fig.26. Influence of power index $n$ on velocity profile at $\tau=4.71$ for sine oscillation.

CASE (b): For principle boundary condition $f(0, \tau)=\operatorname{Cos} \tau$

Figures 27 and 28 shows the variation of $f$ at $\tau=3.14$ and $\tau=6.28$ respectively. It is shown in magnified part of figure Fig.27 that there is no shift of phase for non-Newtonian fluids and the steadiness of $f$ for $n=0.75$ is delayed than for $n=0.5$ and $n=1.5$. It is observed from Fig.28 that as the power index $n$ increases the time required to get steady is also increases it means increase in $n$ reflects the delay the oscillatory boundary effect. The steadiness of velocity comes earlier for $n=0.75$ than $n=0.5$ and $n=1.5$. 


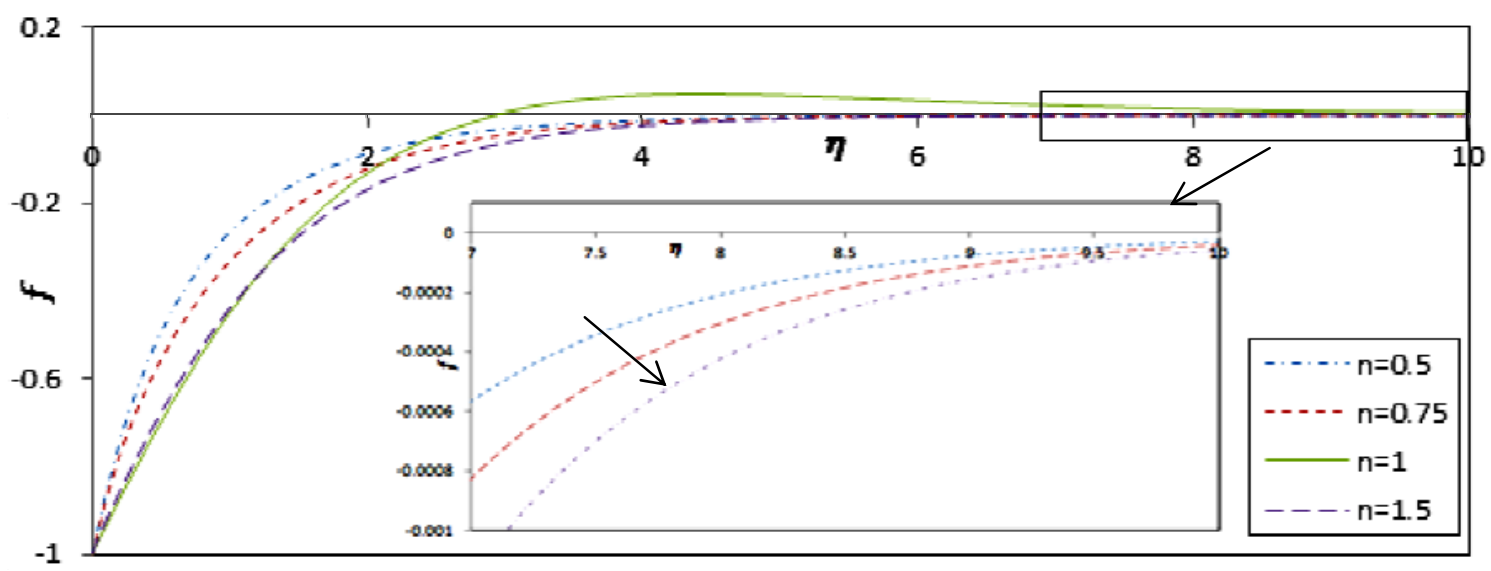

Fig.27. Influence of power index $n$ on velocity profile at $\tau=3.14$ for cosine oscillation.

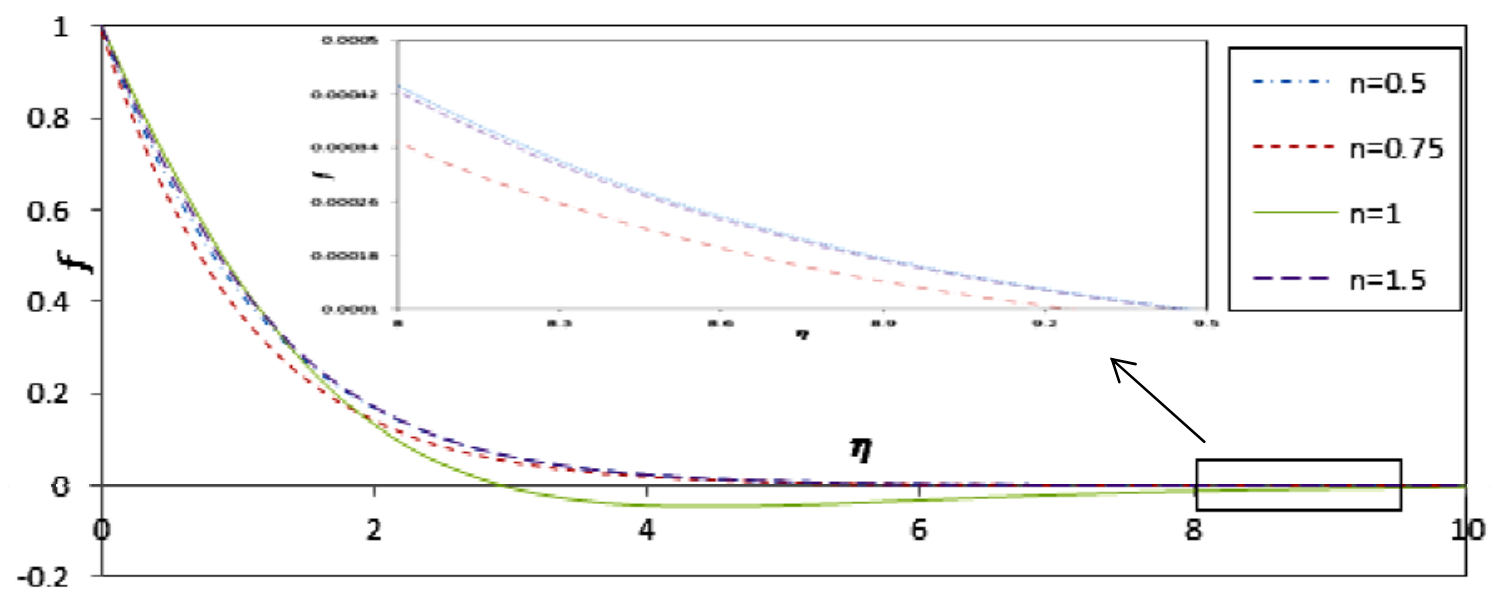

Fig.28. Effect of power index $n$ on velocity profile at $\tau=6.28$ for cosine oscillation.

\subsection{Impact of power index $n$ over the wall shear stress $\tau_{w}$}

Based on the $\tau_{w}$ for power index $n=0.5,0.75$ and $n=1.5$, the control of $n$ over $\tau_{w}$ is shown in Figs 29 and 30 for sine and cosine oscillation at $\tau=5$ and $\tau=3$ respectively for $c_{0}=-0.1$.

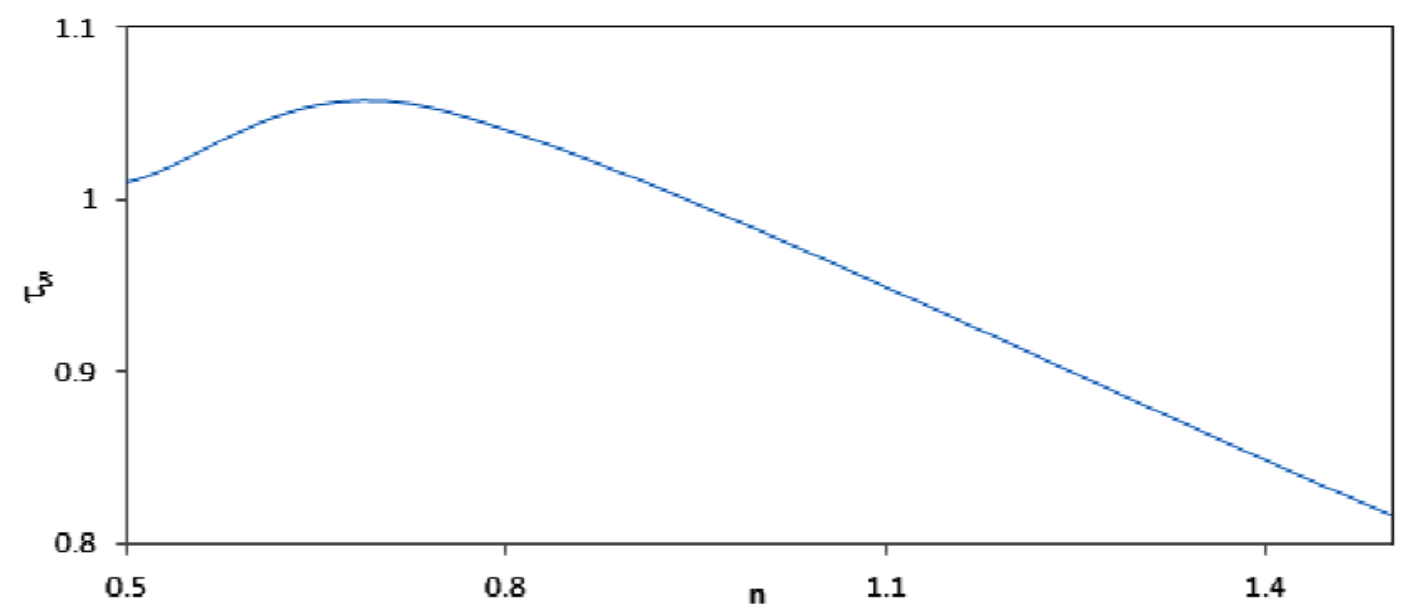

Fig.29.Variation between $\tau_{w}$ and $n$ at $\tau=5$ and $c_{0}=-0.1$ for sine oscillation. 


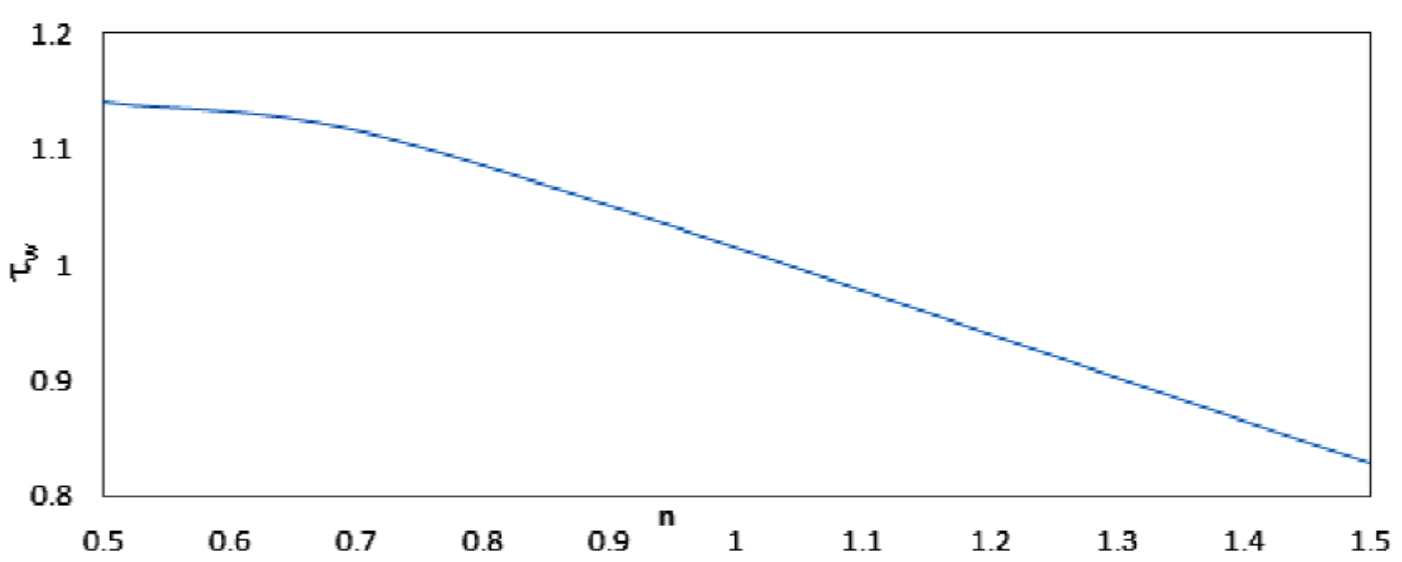

Fig.30. Variation between $\tau_{w}$ and $n$ at $\tau=3$ and $c_{0}=-0.1$ for cosine oscillation.

\subsection{Impact of $n$ for fixed $\eta$}

The variation between forth order approximation of velocity $f$ and time $\tau$ for fixed $\eta$ and $c_{0}$ for different $n$ is discussed below:

The impact of $n$ over $f$ for fixed $\eta=1$ is shown in Figs 31 and 32 for sine and cosine oscillation, respectively. $c_{0}=0.2$ and $c_{0}=-0.25$ are taken for sine and cosine oscillation, respectively. Figure 31 explains that velocity has a maximum amplitude at $\tau=1.57$ for $n=0.75$ and a minimum amplitude at $\tau=4.71$ for $n=0.5$ while Fig. 32 shows that velocity has a maximum amplitude at $\tau=0$ for $n=0.5$ and a minimum amplitude at $\tau=3.14$ for $n=1.5$. It is observed that for sine oscillation the amplitude of velocity for $n=1.5$ decays to zero earlier than the shear thinning fluids while for cosine oscillation it decays to zero later than the shear thinning fluids. Also, it is obvious that as $\eta$ increases from 1 onwards the amplitude respectively decreases. Therefore, the amplitude of the fluid moving due to condition $f(0, \tau)=\operatorname{Sin} \tau$ or $f(0, \tau)=\operatorname{Cos} \tau$ over the wall decreases as we go away from the wall. Also, the curves become more disturbed after $\eta=1$.

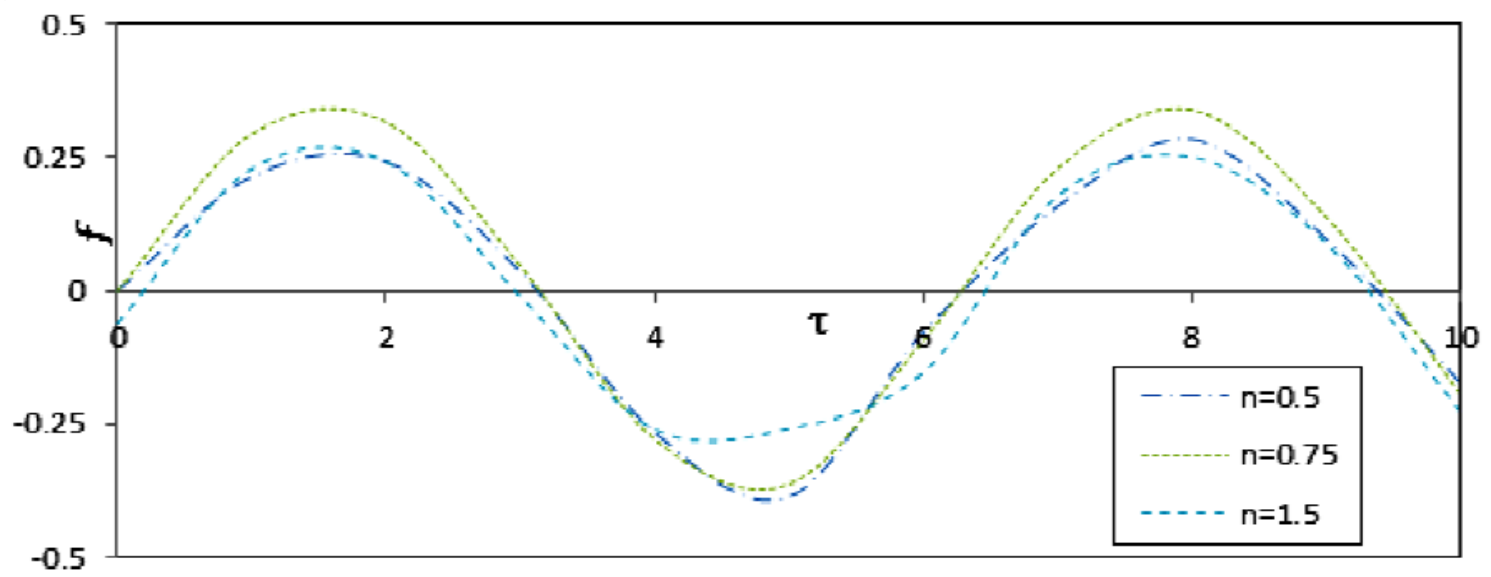

Fig.31. Variation of $4^{\text {th }}$ order approximation of velocity $f$ for different $n$ at $\eta=1$ and $c_{0}=0.2$ for sine oscillation. 


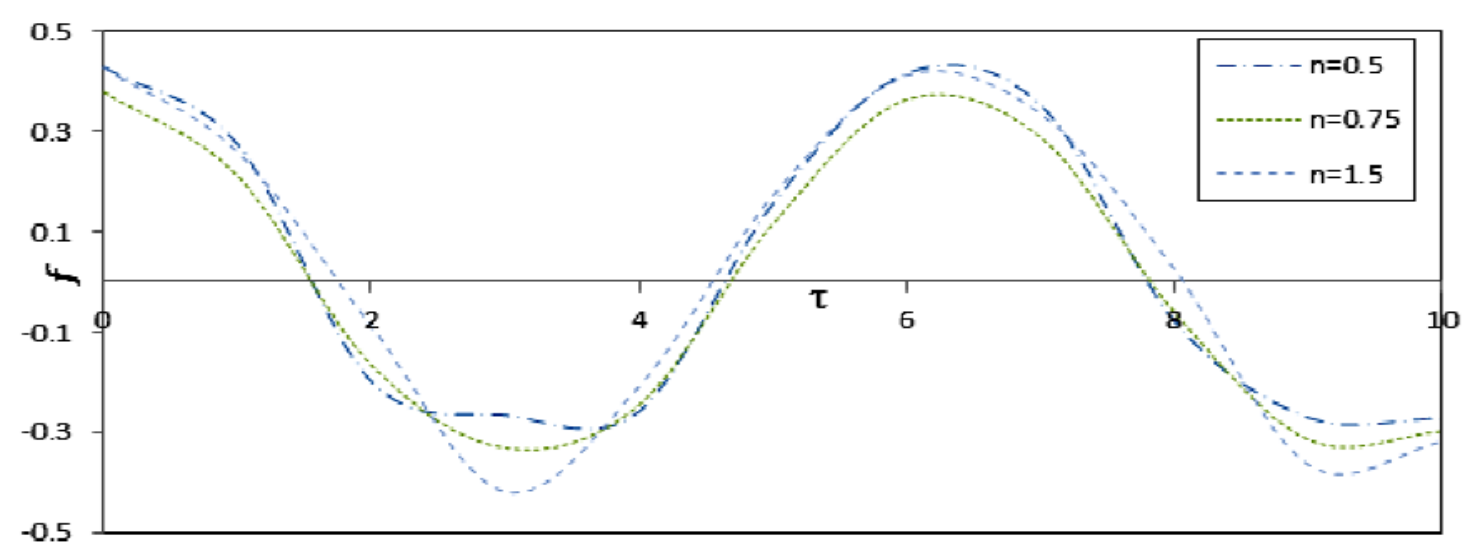

Fig.32. Variation of $4^{\text {th }}$ order approximation of velocity $f$ for different $n$ at $\eta=1$ and $c_{0}=0.2$ for cosine oscillation.

\section{Conclusions}

In this paper, we have studied the velocity field corresponding to the flow of a non-Newtonian Spriggs fluid over a flat plate with oscillatory motion. Homotopy solution for the non-linear problem is obtained and convergence of the series solution is discussed. The plate was subjected to sine and cosine oscillations. The governing equations were non-dimensionalised and solved by using HAM. The influence of transient flow of non-Newtonian Spriggs fluid is greater than that of Newtonian fluid [27]. The impact of non-Newtonian Spriggs fluid over the time requires reaching the steady state for both sine and cosine oscillation of plate is appropriately discussed. Velocity decays to zero faster for a non-Newtonian Spriggs fluid as compared to a Newtonian fluid. Further, oscillations disappear away abruptly for shear thickening fluids than shear thinning fluids. The analyses of wall shear stress for both oscillations are presented through graphs for different time intervals. Both oscillations show similar amplitudes but have phase shift. The action of power index over velocity profile at fixed time is shown. The action of index $n$ on wall shear stress is explored. At a fixed distance from the wall of the plate, the behaviour of sine and cosine oscillations is examined in Sec. 4.6 for shear thinning and shear thickening fluids.

\section{Nomenclature}

$c_{0}-$ convergence control parameter

$f^{\prime}$ - derivative of $f$

$f_{m}-\mathrm{m}^{\text {th }}$ order deformation derivative

$f_{m} *-$ solution of $\mathrm{m}^{\text {th }}$ order deformation

$f_{0} \quad$ - initial guess

$f(\eta)$ - non-dimensional velocity

$\mathcal{L}-$ auxiliary linear operator

$\mathcal{L}^{-1}$ - inverse linear operator

$\mathcal{N}$ - non-linear operator

$n$ - power index

$p$ - pressure

$t$ - time

$U$ - reference velocity

$u$ - velocity component in $x$-direction

$q$ - embedding parameter

$v$ - velocity component in $y$-direction

$\dot{\gamma}$ - shear rate 


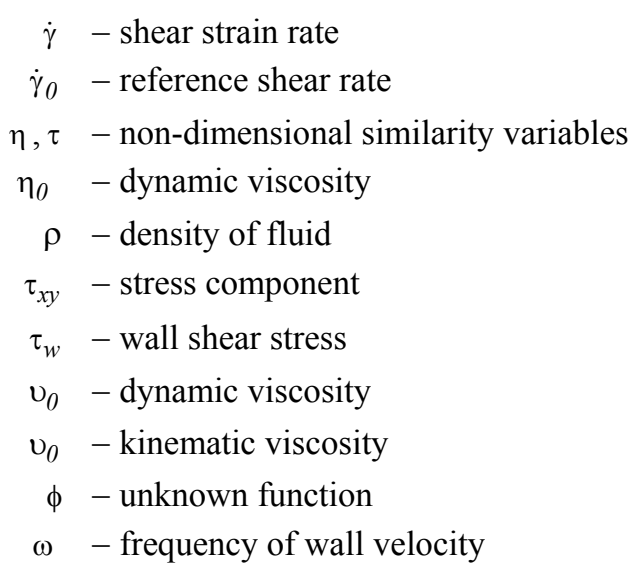

\section{References}

[1] Chhabra R.P. and Richardson J. (2008): Non-Newtonian Flow and Applied Rheology: Engineering Applications (second ed). - Oxford: Butterworth-Heinemann.

[2] Irgens F. (2014): Rheology and non-Newtonian Fluids. - New York: Springer Int. Publ..

[3] Arada N. and Pires M. (2007): Viscosity effects on flows of generalized Newtonian fluids through curved pipes. Comput. Math. with Appl., vol.53, pp.625-646.

[4] Bair S and Qureshi F. (2003): The generalized Newtonian fluid model and elastohydrodynamic film thickness. - J. Tribol., vol.125, pp.70.

[5] Spriggs T. (1965): A four-constant model for viscoelastic fluids. - Chem. Eng. Sci., vol.20, pp.931-940.

[6] Lavrov A. (2015): Flow of truncated power-law fluid between parallel walls for hydraulic fracturing applications. - J. Nonnewton. Fluid Mech., vol.223, pp.141-146.

[7] Greenwood J.A. and Kauzlarich J.J. (2015): Elastohydrodynamic film thickness for shear- thinning lubricants. Proc. Inst. Mech. Eng. Part J. Jouranal Eng., vol.212, pp.179-191.

[8] Adusmilli R.S. and Hill G.A. (1984): Transient laminar flows of truncated power law fluids in pipes. - Can. J. Chem. Eng., vol.62, pp.594-601.

[9] Raju C.S.K. and Sandeep N. (2016): Heat and mass transfer in MHD non-Newtonian bio-convection flow over a rotating cone/plate with cross diffusion. - J. Mol. Liq., vol.215, pp.115-126.

[10] Rashidi M.M., Bagheri S., Momoniat E. and Freidoonimehr N. (2017): Entropy analysis of convective MHD flow of third grade non-Newtonian fluid over a stretching sheet. - Ain Shams Eng. J., vol.8, pp.77-85.

[11] Mohyud-din S.T., Khan U., Ahmed N. and Rashidi M.M. (2017): Stokes first problem for MHD flow of Casson nanofluid. - Multidiscip. Model. Mater. Struct., vol.13, pp.2-10.

[12] Gorla R.S.R. and Vasu B. (2016): Unsteady convective heat transfer to a stretching. - J. Nanofluid, vol.5, pp.1-14.

[13] Gorla R.S.R., Vasu B. and Siddiqa S. (2016): Transient combined convective heat transfer over a stretching surface in a non-Newtonian nanofluid using Buongiorno's model. - J. Appl. Math. Phys., vol.4, pp.443-460.

[14] Christov I.C. (2010): Stokes first problem for some non-Newtonian fluids: Results and mistakes. - Mech. Res. Commun., vol.37, pp.717-723.

[15] Stokes G.G. (1851): On the effect of internal friction of fluids on the motion of pendulums. - Math. Phys. Pap., vol.3, pp.1880-1905.

[16] Ezzat M.A., El-bary A.A. and Ezzat S.M. (2013): Stokes first problem for a thermoelectric Newtonian fluid. Meccanica, vol.48, pp.1161-1175.

[17] Liu W., Peng J. and Zhu K. (2016): Finite depth Stokes' first problem of thixotropic fluid. - Appl. Math. Mech., vol.37, pp.59-74.

[18] Zaman H., Ubaidullah, Shah M.A. and Ibrahim M. (2014): Stokes first problem for an unsteady MHD fourth- 
grade fluid in a non-porous half space with Hall currents. - IOSR J. Appl. Phys., vol.6, pp.7-14.

[19] Rajagopal K.R. (1982): A note on unsteady undirectional flows of a non-Newtonian fluid. - Int. J. Non. Linear. Mech., vol.17, pp.369-373.

[20] Ishfaq N., Khan W.A. and Khan Z.H. (2017): The Stokes' second problem for nanofluids. - J. King Saud Univ. Sci., pp.1-5.

[21] Duan J. and Qiu X. (2014): The periodic solution of Stokes' second problem for viscoelastic fluids as characterized by a fractional constitutive equation. - J. Nonnewton. Fluid Mech., vol.205, pp.11-15.

[22] Farkhadnia F., Kamrani R. and Ganji D.D. (2014): Analytical investigation for fluid behavior over a flat plate with oscillating motion and wall transpiration. - New Trends Math. Sci., vol.2, pp.178-189.

[23] Ali F., Norzieha M., Sharidan S., Khan I. and Hayat T. (2012): New exact solutions of Stokes' second problem for an MHD second grade fluid in a porous space. - Int. J. Non. Linear. Mech., vol.47, pp.521-525.

[24] Asghar S., Nadeem S., Hanif K. and Hayat T. (2006): Analytic solution of Stokes second problem for secondgrade fluid. - Math. Probl. Eng., vol.2006, pp.1-8.

[25] Fetecau C., Jamil M., Fetecau C. and Siddique I. (2009): A note on the second problem of Stokes for Maxwell fluids. - Int. J. Non. Linear. Mech., vol.44, pp.1085-1090.

[26] Ai L. and Vafai K. (2005): An investigation of Stokes'second problem for non-Newtonian fluids. - Numer. Heat Transf. Part A Appl., vol.47, pp.955-980.

[27] Erdogan M.E. (2000): A note on an unsteady flow of a viscous fluid due to an oscillating plane wall. - Int. J. Non. Linear. Mech., vol.35, pp.1-6.

[28] Holmes M.H. (2013): Introduction to Perturbation Methods. - Springer New York Heidelberg Dordrecht London.

[29] Bhimsen S. (2012): Perturbation Methods for Differential Equations. - Springer Sci. Bus. Media..

[30] Liao S. (2014): Advances in the Homotopy Analysis Method. - World Scientific.

[31] Rashidi M.M., Ashraf M., Rostami B., Rastegari M.T. and Bashir S. (2016): Mixed convection boundary - layer flow of a micro polar fluid towards a heated shrinking sheet by Homoptopy Analysis Method. - Theraml Sci., vol. 20, pp.21-34.

[32] Hayat T., Qayyum S., Alsaedi A. and Ahmad B. (2017): Nonlinear convective flow with variable thermal conductivity and Cattaneo-Christov heat flux. - Neural Comput. Appl., pp.1-11.

[33] Das D., Ray P.C. and Bera R.K. (2016): Solution of Nonlinear Fractional Differential Equation (NFDE) by four different approximate methods. - Int. J. Sci. Res. Educ., vol.4, pp.5598-5620.

[34] Al-Shara S., Awawdeh F. and Abbasbandy S. (2017): An automatic scheme on the Homotopy analysis method for solving non-linear algebraic equations. - Ital. J. Pure Appl. Math., vol.37, pp.5-14.

[35] Zhong X. and Liao S. (2017): Analytic Solutions of Von Karman plate under arbitrary uniform pressure (I): Equations in differential form analytic solutions of Von Karman plate under arbitrary uniform pressure Part I: Equations in differential form. - Stud. Appl. Math., vol.138, pp.371-400.

[36] Turkyilmazoglu M. (2010): A note on the homotopy analysis method. - Appl. Math. Lett., vol.23, pp.1226-1230.

[37] Sajid M. and Hayat T. (2009): Comparison of HAM and HPM solutions in heat radiation equations. - Int. Commun. Heat Mass Transfer, vol.36, pp.59-62.

[38] Marinca V., Ene R.D. and Marinca B. (2014): Analytic approximate solution for Falkner-Skan equation. - Sci. World J., vol.2014.

[39] Liao S. (1999): An explicit, totally analytic approximate solution for Blasius' viscous flow problems. - Int. J. Non. Linear. Mech., vol.34, pp.759-778.

[40] Currie I.G. (2012): Fundamental Mechanics of Fluids. - CRC Press. 